\title{
Newtonian and Non-Newtonian Fluids through Permeable Boundaries
}

\author{
Riëtte Maritz and Emile Franc Doungmo Goufo \\ Department of Mathematical Sciences, University of South Africa, P.O. Box 392, Pretoria 0003, South Africa \\ Correspondence should be addressed to Emile Franc Doungmo Goufo; dgoufef@unisa.ac.za
}

Received 20 August 2014; Accepted 17 September 2014; Published 1 October 2014

Academic Editor: Abdon Atangana

Copyright (C) 2014 R. Maritz and E. F. Doungmo Goufo. This is an open access article distributed under the Creative Commons Attribution License, which permits unrestricted use, distribution, and reproduction in any medium, provided the original work is properly cited.

\begin{abstract}
We considered the situation where a container with a permeable boundary is immersed in a larger body of fluid of the same kind. In this paper, we found mathematical expressions at the permeable interface $\Gamma$ of a domain $\Omega$, where $\Omega \subset \mathfrak{R}^{3}$. $\Gamma$ is defined as a smooth two-dimensional (at least class $C^{2}$ ) manifold in $\Omega$. The Sennet-Frenet formulas for curves without torsion were employed to find the expressions on the interface $\Gamma$. We modelled the flow of Newtonian as well as non-Newtonian fluids through permeable boundaries which results in nonhomogeneous dynamic and kinematic boundary conditions. The flow is assumed to flow through the boundary only in the direction of the outer normal $\mathbf{n}$, where the tangential components are assumed to be zero. These conditions take into account certain assumptions made on the curvature of the boundary regarding the surface density and the shape of $\Omega$; namely, that the curvature is constrained in a certain way. Stability of the rest state and uniqueness are proved for a special case where a "shear flow" is assumed.
\end{abstract}

\section{Introduction}

The flow of incompressible Navier-Stokes fluids and fluids of second grade through permeable boundaries and past porous walls has been studied under various conditions. The equation of motion for incompressible flows in Newtonian fluids (Navier-Stokes equations) under no-slip boundary conditions has been studied extensively from many perspectives. Since the pioneering papers of Leray [1-3] and Hopf [4] questions of the existence, stability $[5,6]$, and uniqueness of both classical and weak solutions have received more than their fair share of attention.

Recently the same issues have been studied for nonNewtonian fluids of second grade. The studies cover both weak solutions [7-12] and classical solutions for homogeneous Dirichlet boundary data [13] and nonhomogeneous boundary data $[6,14,15]$.

Unlike Newtonian fluids, fluids of second grade (and other non-Newtonian species) have the property of developing "normal stresses differences" at boundaries. It was shown, for example, by Berker [16] that if an incompressible flow of a fluid of grade two satisfies the homogeneous Dirichlet boundary condition. The stress at the boundary is given by $\mathbf{t}=\left(-p+\alpha|\boldsymbol{\omega}|^{2}\right) \mathbf{n}+\left[\mu \boldsymbol{\omega}+2 \alpha \partial_{t} \boldsymbol{\omega}\right] \wedge \mathbf{n}$, where $\mathbf{n}$ is the unit exterior normal to the boundary and $\omega=\nabla \wedge \mathbf{v}$ is the vorticity. The wedge denotes a vector product. Thus there is a normal component of stress at the boundary in addition to the pressure. The question becomes what governs the flow across the boundary? Possible ways of circumventing this question may be to "prescribe" the normal component of the velocity field at the boundary or to prescribe mass or momentum flux. The prescription of shear stress has also been suggested. ([16, 17]). Nonlinear or non-Newtonian fluids are fluids like molten metals, multigrade oils, printing inks, paints, suspensions, polymer solutions, molten plastics, blood, protein solutions, and ice [18]. These fluids cannot be described by the above model. The study of these interesting substances has proved to be very important with the growth of the polymer and plastics industry over the last four decades. Consequently, an interest has arisen to study the flow of these nonlinear fluids and, in the case of this model, second-grade fluids, through permeable boundaries. The boundary conditions alone in such circumstances are an interesting topic for study. Works by Berker [16] and Rajagopal and Gupta [19] can be mentioned in this regard. 
In this study we shall provide an alternative approach through the formulation of "dynamics at the boundary," the idea being that the normal component of velocity at the boundary is viewed as an unknown function which satisfies a differential equation intricately coupled to the flow in the region "enclosed" by the boundary.

A glimpse of the history of the research on nonNewtonian and Newtonian fluids around porous boundaries is given in Section 2. Notation and definitions precede Sections 4 and 5 which deal with the constitutive equations and the modelling of permeability. In Section 6 the expressions on the interface $\Gamma$ are given. The alternative model is studied and the stability and uniqueness are proved in Section 7. Section 8 concludes the study and further explorations are discussed.

\section{Backgroud}

Berker [16] studied the two-dimensional creeping flow of a second-order fluid with nonparallel porous walls. An additional velocity boundary condition was needed. The other conditions they used were due to the usual no-slip conditions. This additional velocity boundary condition was to prescribe the rate of shear at the wall. The problem was then solved numerically by a standard routine.

In 1989 Rajagopal and Kaloni [20] wrote remarks on boundary conditions for flows of fluids of the differential type. Rajagopal [21] discusses a lot of related issues. Rajagopal and Gupta [19] studied the flow of an incompressible fluid of second grade past an infinite porous plate subjected to either suction or blowing at the plate. They studied fluids modelled by

$$
\mathbf{T}=-p \mathbf{I}+\mu \mathbf{A}+\alpha_{1}\left[D_{t} \mathbf{A}+\mathbf{A}(\nabla \mathbf{v})+(\nabla \mathbf{v})^{T} \mathbf{A}\right]+\alpha_{2} \mathbf{A}^{2} .
$$

No assumptions were made about the material moduli $\alpha_{1}$ and $\alpha_{2}$. For the boundary value problem they considered, it was found that the velocity distributions do not depend on the normal stress modulus $\alpha_{2}$, but the pressure does. They found that it was possible to produce an exact solution which is asymptotic in nature for both "suction" and "blowing" at the plate if the material modulus $\alpha_{1}>0$. For $\alpha_{1}<0$, they found that such solutions could not exist in the case of blowing, a result which was in keeping with the classical incompressible fluid. Fosdick and Rajagopal [22] have shown that the model (1) whose material modulus $\alpha_{1}<0$ exhibits anomalous behaviour was not to be expected of any fluid of rheological interest (also see [23]). Proudman studied an example of steady laminar flow at a large Reynolds number [24].

Beavers and Joseph [25] studied the flow of a Newtonian fluid over a porous surface in 1967. They found that if the governing differential system was not to be underdetermined, it was necessary to specify some condition on the tangential component of the velocity of the free fluid at the porous interface. It is usual in these analyses to approximate the fluid motion near the true boundary with an adherence condition for the tangential component of velocity of the free fluid at some boundary. Because of a certain ambiguity which is implied by the notion of a "true" boundary for a permeable material, it was found useful to define a nominal boundary. They fixed a nominal boundary by first defining a smooth geometric surface and then assuming that the outermost perimeters of all the surface pores of the permeable material are in this surface. Thus, if the surface pores were filled with solid material to the level of their respective perimeters, a smooth impermeable boundary of the assumed shape would result. This definition is precise when the geometry is simple (planes, spheres, cylinders, etc.) but may not be fully adequate in more complex situations. Beavers and Joseph's [25] experiment was designed to examine the tangential flow in the boundary region of a permeable interface. The results of this experiment indicate that the effects of viscous shear appear to penetrate into the permeable material in a boundary layer region, producing a velocity distribution similar to that depicted in the following figure. The tangential component of the velocity of the free fluid at the porous boundary can be considerably greater than the mean filter velocity within the body of the porous material.

In Figure 1 the plane $y=0$ defines a nominal surface for the permeable material. The flow through the body of the permeable material, which is homogeneous and isotropic, is assumed to be governed by Darcy's Law. Read more of the status on Darcy's Law in [26]. In the absence of body forces Darcy's Law may be written as $Q=-(k / \mu)(d P / d x)$, where $k$ is the "permeability" of the material and $Q$ is the volume flow rate per unit of the cross-sectional area. As such, $Q$ represents the filter velocity rather than the true velocity of the fluid in the pores. The measured pressure gradient is denoted by $d P / d x$.

\section{Basic Notation}

We work in Euclidean 3 space. The following notation will be used throughout:

$$
\begin{aligned}
|\mathbf{x}| & :=\sqrt{\sum_{1}^{3} x_{i}^{2}} \text { denotes the Euclidean norm. } \\
\partial_{i} & :=\frac{\partial}{\partial x_{i}} ; \quad i=1,2,3 . \\
\partial_{t} & :=\frac{\partial}{\partial t} . \\
{[\nabla p]_{i} } & :=\partial_{i} p \quad \text { if } p \text { is a scalar field. } \\
{[\nabla \mathbf{v}]_{i j} } & :=\partial_{j} v_{i} ; \quad i, j=1,2,3, \text { if } \mathbf{v} \text { is a vector field. } \\
{[\nabla \mathbf{v}]_{i j}^{T} } & :=\partial_{i} v_{j} ; \quad i, j=1,2,3, \text { if } \mathbf{v} \text { is a vector field. } \\
\nabla \cdot \mathbf{v} & :=\sum_{i=1}^{3} \partial_{i} v_{i} \quad \text { if } \mathbf{v} \text { is a vector field. } \\
\mathbf{v} \cdot \nabla & :=\sum_{i=1}^{3} v_{i} \partial_{i} \quad \text { if } \mathbf{v} \text { is a vector field. }
\end{aligned}
$$




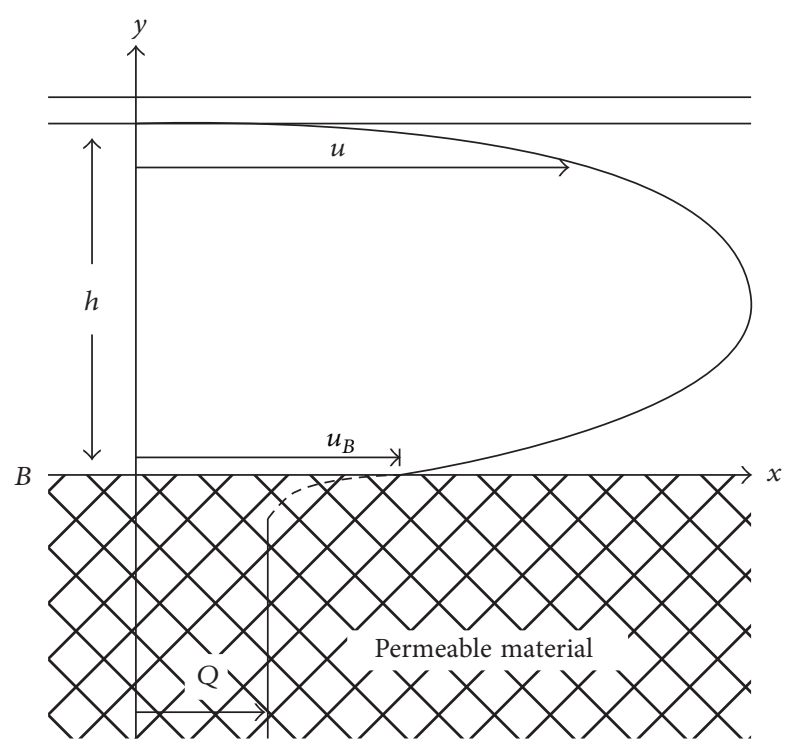

FIGURE 1: Velocity profile for the rectilinear flow in a horizontal channel formed by a permeable lower wall $(y=0)$ and an impermeable upper wall $(y=h)$.

$$
[\nabla \cdot \mathbf{T}]_{j}:=\sum_{i=1}^{3} \partial_{i} T_{i j} ; \quad j=1,2,3,
$$

if $\mathbf{T}$ is a matrix (tensor) with

Euclidean components $T_{i j}$.

$[\mathbf{v} \otimes \mathbf{v}]_{i j}:=v_{i} v_{j} ; \quad i, j=1,2,3$, if $\mathbf{v}$ is a vector.

$D_{t}:=\partial_{t}+\mathbf{v} \cdot \nabla ; \quad D_{t}$ is the material time derivative.

$\mathbf{v} \wedge \mathbf{u}:=$ denotes the usual vector

product of the vectors $\mathbf{v}$ and $\mathbf{u}$

$\nabla \wedge \mathbf{v}:=\operatorname{curl} \mathbf{v}$

If $\mathbf{A}$ and $\mathbf{B}$ are second order tensors we shall use the notations $\mathbf{A}: \mathbf{B}=\sum_{i, j=1}^{3} A_{i j} B_{i j}$ and $|\mathbf{A}|^{2}=\mathbf{A}: \mathbf{A}$. Let $\Omega \subset \mathbf{R}^{3}$ be a bounded domain with a smooth (at least $\mathbf{C}^{2}$ ) boundary $\Gamma$. Let $\mathbf{n}=\mathbf{n}(x)$ denote the unit exterior normal to $\Gamma$ at $x$. We shall be concerned with smooth vector fields $\mathbf{v}=\mathbf{v}(x)$ defined in $\Omega$ such that on $\Gamma$ it has the form $\gamma_{o} \mathbf{v}(x)=-\eta(x) \mathbf{n}(x)$, where $\gamma_{o}$ is the trace operator denoting boundary values and $\eta$ is a smooth scalar field defined on $\Gamma$. Associated with $\nabla \mathbf{v}$ we define the symmetric and skew-symmetric tensors $\mathbf{A}$ and $\mathbf{W}$ as $\mathbf{A}=\mathbf{A}(\mathbf{v})=\nabla \mathbf{v}+(\nabla \mathbf{v})^{T}$ and $\mathbf{W}=\mathbf{W}(\mathbf{v})=\nabla \mathbf{v}-(\nabla \mathbf{v})^{T}$, where $(\nabla \mathbf{v})^{T}$ denotes the transpose of the gradient of $\mathbf{v}$. The rate of deformation tensor is related to $\mathbf{A}$ by $\mathbf{D}(\mathbf{v})=(1 / 2) \mathbf{A}(\mathbf{v})$. We note that if $\mathbf{v}$ is solenoidal $(\nabla \cdot \mathbf{v}=0)$ then trace $\mathbf{A}(\mathbf{v})=2 \nabla \cdot \mathbf{v}=$ 0 and, for any vector $\mathbf{a}, \mathbf{W}(\mathbf{v}) \mathbf{a}=\boldsymbol{\omega} \wedge \mathbf{a}$, where $\boldsymbol{\omega}=\nabla \wedge \mathbf{v}$ denotes the vorticity associated with $\mathbf{v}$.

\section{The Constitutive Equations}

The stress tensor for the linear viscous Newtonian model is $\mathbf{T}=-p \mathbf{I}+\mu\left(\nabla \mathbf{v}+(\nabla \mathbf{v})^{T}\right)$, with $p$ as the pressure, $\mu$ as the coefficient of viscosity, and $\mathbf{v}$ as the velocity of the fluid. This model describes the flow of fluids like water and other similar fluids. Lamb [27] and Ladyzhenskaya [28] wrote mathematical theories on viscous incompressible flow.

Fluids of a differential type [29-31], of which RivlinEricksen fluids are a subclass, are depicted by a popular nonlinear model. Fluids of complexity $n$ form an important subclass of the fluids of a differential type. For incompressible fluids of complexity $n$ the Cauchy stress tensor is of the form $\mathbf{T}=-p \mathbf{I}+\mathbf{F}\left(\mathbf{A}_{1}, \ldots, \mathbf{A}_{n}\right)$. The pressure $p$ is not a thermodynamic variable and the term $-p \mathbf{I}$ reflects Pascal's law, which is inherent to all fluids. $\mathbf{A}_{1}, \ldots, \mathbf{A}_{n}$ are the first $n$ Rivlin-Ericksen tensors [21] defined recursively by

$$
\begin{aligned}
& \mathbf{A}_{1}=\nabla \mathbf{v}+(\nabla \mathbf{v})^{T}=\mathbf{A}, \\
& \mathbf{A}_{n}=D_{t} \mathbf{A}_{n-1}+\mathbf{A}_{n-1}(\nabla \mathbf{v})+(\nabla \mathbf{v})^{T} \mathbf{A}_{n-1}, \quad n \geq 2 .
\end{aligned}
$$

Fluids of grade $n$ are examples of fluids of complexity $n$. The stress tensors for fluids of grades 1 and 2 respectively, are assumed to be of the form

$$
\begin{gathered}
\mathbf{T}^{[1]}=-p \mathbf{I}+\mu \mathbf{A}_{1}, \\
\mathbf{T}^{[2]}=\mathbf{T}^{[1]}+\alpha_{1} \mathbf{A}_{2}+\alpha_{2} \mathbf{A}_{1}^{2},
\end{gathered}
$$

where $\mu$ and $\alpha_{i}$ are material coefficients (possibly temperature-dependent).

For incompressible fluids of second grade, the stressdeformation relation then becomes

$$
\begin{aligned}
\mathbf{T}=\mathbf{T}^{[2]}= & -p \mathbf{I}+\mu \mathbf{A}+\alpha_{1} D_{t} \mathbf{A} \\
& +\alpha_{1}\left(\mathbf{A} \nabla \mathbf{v}+(\nabla \mathbf{v})^{T} \mathbf{A}\right)+\alpha_{2} \mathbf{A}^{2},
\end{aligned}
$$

where $p$ and $\mathbf{v}$ are the pressure and the velocity fields. Here $\mu$ is the coefficient of viscosity and $\alpha_{1}$ and $\alpha_{2}$ are material coefficients or "normal stress moduli." In this case $\mathbf{A}=\mathbf{A}_{1}$.

To use the relation (5) for the modelling of a fluid, the fluid has to be compatible with thermodynamics in the sense that all flows of the fluid must satisfy the Clausius-Duhem inequality, and the assumption must be made that the specific Helmholtz free energy is at a minimum when the fluid is in equilibrium. Under these assumptions, $\alpha_{1}$ and $\alpha_{2}$ [32] must satisfy

$$
\alpha_{1}+\alpha_{2}=0
$$

Considerations of stability of the rest state require the assumptions $\mu$ and $\alpha_{1}$ to be nonnegative; that is, $\mu>0$, $\alpha_{1}>0$. See [32]. Under assumption (6), which we shall follow throughout, the form of the stress tensor $\mathbf{T}$ given in (5) reduces to a more compact expression. To obtain this we note that $\nabla \mathbf{v}=(1 / 2)(\mathbf{A}+\mathbf{W})$ and $(\nabla \mathbf{v})^{T}=(1 / 2)(\mathbf{A}-\mathbf{W})$, so that

$$
\begin{aligned}
\alpha_{1}\left(\mathbf{A} \nabla \mathbf{v}+(\nabla \mathbf{v})^{T} \mathbf{A}\right) & =\frac{\alpha_{1}}{2}[\mathbf{A}(\mathbf{A}+\mathbf{W})+(\mathbf{A}-\mathbf{W}) \mathbf{A}] \\
& =\alpha_{1} \mathbf{A}^{2}+\frac{\alpha_{1}}{2}(\mathbf{A W}-\mathbf{W A}) .
\end{aligned}
$$




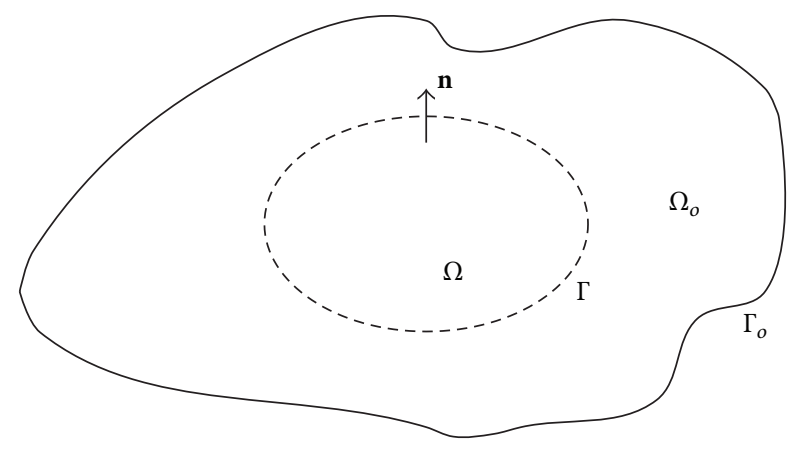

FIGURE 2: Profile for normal flow through the permeable wall $\Gamma$.

Therefore, by (5) and (7)

$$
\mathbf{T}=-p \mathbf{I}+\mu \mathbf{A}+\alpha D_{t} \mathbf{A}+\frac{\alpha}{2}(\mathbf{A W}-\mathbf{W A}),
$$

where we have set $\alpha_{1}=\alpha$.

Remark 1. Please note that for the Navier-Stokes equations we take $\alpha=0$ [33].

\section{Modelling of Permeability}

We study the motion of fluids around and through a fixed porous container filled with the same fluid. The interior of the porous container is an open bounded set $\Omega \subset \mathbf{R}^{3}$ and the porous boundary, $\Gamma$, is smooth. The surrounding fluid domain, $\Omega_{o}$, is bounded and its outer boundary is denoted by $\Gamma_{o}$. The exterior normal to $\Omega$ on $\Gamma$ is denoted by $\mathbf{n}$. Figure 2 illustrates the situation where the curvature of the boundary $\Gamma$ of $\Omega$ is nonnegative.

Permeability of the walls of the container is described by assuming that at the boundary $\Gamma$ the flow $\mathbf{v}$ has the direction of the normal:

$$
\gamma_{o} \mathbf{v}(x, t)=-\eta(x, t) \mathbf{n}(x) .
$$

The velocity component $\eta$ is treated as an unknown and an evolution equation has to be found for it. We model the surface $\Gamma$ as having an effective area measure $d a$ which has a density function $\zeta(x)$ with respect to the area measure $d s$. Thus $d a=\zeta(x) d s$. The effective area through which fluid can permeate is not more than the surface area and therefore $0 \leq \zeta(x) \leq 1$ for any $x \in \Gamma$. If $\zeta(x) \equiv 0$, the wall is impermeable and if $\zeta(x) \equiv 1$, there is no wall.

In order to obtain expressions for mass and momentum in a boundary patch $\Gamma^{\prime}$, we let the patch be heuristically represented by a volume $G$ built from copies of $\Gamma^{\prime}$ (Figure 3). This is in line with the Beavers-Joseph thinking which was discussed before. For this volume we set up a coordinate system consisting of a "radial part" $r$, which has the direction of the normal vector $\mathbf{n}$, and a "surface part" made up by vectors tangential to $\Gamma^{\prime}$. For the mass of $G$ we obtain

$$
\int_{G} \rho d x=\int_{\Gamma^{\prime}} \int_{0}^{\delta} \rho d r d a=\int_{\Gamma^{\prime}} \int_{0}^{\delta} \rho d r \zeta d s=\int_{\Gamma^{\prime}} \rho \zeta \delta d s,
$$

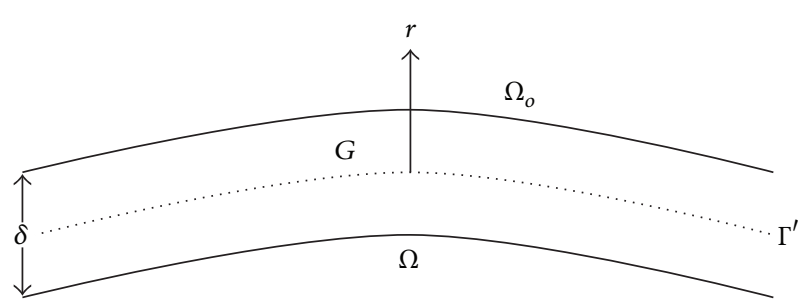

Figure 3: Heuristics of the permeable boundary.

where $\delta$ is some measure of thickness. With the aid of these concepts we introduce the surface density of the fluid at $x \in \Gamma$ as

$$
\sigma(x)=\delta(x) \zeta(x) \rho,
$$

where $\rho$ is the volume density of the fluid.

To obtain the equation of motion for fluid in the boundary, we assume that the rate of change of linear momentum in the boundary is explained by stress forces at both sides of the boundary.

Let $\mathbf{T}$ and $\mathbf{T}^{\prime}$ denote the stress tensors at the sides of the boundary facing $\Omega$ and $\Omega_{o}$, respectively, and let $\mathbf{P}$ and $\mathbf{P}^{\prime}$ denote the transfer-of-momentum tensors on the two sides. On an arbitrary boundary patch $\Gamma^{\prime} \subset \Gamma$ the law of conservation of linear momentum is stated in the following way:

$$
\begin{aligned}
\partial_{t} \int_{\Gamma^{\prime}} \sigma(x) \gamma_{o} \mathbf{v} d s= & \int_{\Gamma^{\prime}}\left[\mathbf{P n}-\mathbf{P}^{\prime} \mathbf{n}\right] d a \\
& +\int_{\Gamma^{\prime}}\left[-\mathbf{T}^{\prime}(-\mathbf{n})-\mathbf{T n}\right] d s
\end{aligned}
$$

with $\sigma$ as defined in (11), and it follows that

$$
\sigma(x) \partial_{t} \gamma_{o} \mathbf{v}-\zeta\left[\mathbf{P}-\mathbf{P}^{\prime}\right] \mathbf{n}=\left[\mathbf{T}^{\prime}-\mathbf{T}\right] \mathbf{n} .
$$

From (13) we have $\sigma(x) \partial_{t} \eta(x, t)+\zeta \mathbf{n} \cdot\left[\mathbf{P}-\mathbf{P}^{\prime}\right] \mathbf{n}=\mathbf{n} \cdot$ $\mathbf{T n}-\mathbf{n} \cdot \mathbf{T}^{\prime} \mathbf{n}$. In the domain $\Omega$ the momentum flux tensor is given by $\mathbf{P}=\rho \mathbf{v} \otimes \mathbf{v}$. In accordance with this, we shall take $\mathbf{P}=\rho \eta^{2} \mathbf{n} \otimes \mathbf{n}$ at the boundary. The tensor $\mathbf{P}^{\prime}$ will be taken as zero.

We take $\mathbf{T}^{\prime}=\ell \mathbf{I}$ to obtain from (13)

$$
\sigma(x) \partial_{t} \eta+\zeta \rho \eta^{2}=\mathbf{n} \cdot \mathbf{T n}-\ell(t) .
$$

From the incompressibility of the flow in $\Omega$ it follows that

$$
-\int_{\Gamma} \eta d s=0 .
$$

\section{Expressions at the Interface}

In order to obtain expressions for the stress tensors $\mathbf{T}$ and $\mathbf{T}^{\prime}$ as well as the acceleration at the boundary through which only normal flow occurs, we obtain a formal expression for the symmetric tensor $\mathbf{A}$ on a surface which is immersed in fluid. We shall eventually use these expressions in postulating 
the form of $\mathbf{T}$ and $\mathbf{T}^{\prime}$ and in formulating a boundary condition which expresses zero tangential acceleration at a wall.

We consider a smooth vector field $\mathbf{v}(\mathbf{x})$ defined on a domain $\Omega \subset \mathbf{R}^{3}$ and a smooth two-dimensional (at least class $C^{2}$ ) manifold $\Gamma \subset \Omega$ so that $\mathbf{v}$ and $\nabla \mathbf{v}$ are defined on $\Gamma$. Let $\mathbf{n}(\mathbf{x})$ be the unit normal to $\Gamma$ at the point $\mathbf{x} \in \Gamma$.

At any point $\mathbf{x}$ on $\Gamma$ we consider two orthogonal curves $c_{1}$ and $c_{2}$ in a neighbourhood of $x$ parametrised by arc lengths $s_{1}$ and $s_{2}$, respectively. Let $\boldsymbol{\tau}_{\mathbf{1}}$ and $\boldsymbol{\tau}_{\mathbf{2}}$ be the unit tangents to the principal normal curves at a point on the surface. For local coordinates we use the orthogonal system formed by $\boldsymbol{\tau}_{1}, \boldsymbol{\tau}_{2}$, and $\mathbf{n}$. Under the convention that $\boldsymbol{\tau}_{\mathbf{1}} \wedge \boldsymbol{\tau}_{\mathbf{2}}=\mathbf{n}$ we have $\mathbf{n} \wedge \boldsymbol{\tau}_{1}=$ $\boldsymbol{\tau}_{2}$ and $\mathbf{n} \wedge \boldsymbol{\tau}_{2}=-\boldsymbol{\tau}_{1}$. Let $\kappa_{1}$ and $\kappa_{2}$ represent the principal curvatures at a point on the surface and let $K=\kappa_{1}+\kappa_{2}$ denote the mean curvature.

\section{Assumptions}

(1) We shall assume throughout that the surface density is bounded and bounded away from zero; that is, there exist constants $s$ and $S$ such that

$$
0<s \leq \sigma(x) \leq S \quad \forall x \in \Gamma .
$$

Also, we assume $\sigma \in C^{\infty}(\Gamma)$.

(2) Apart from the smoothness of $\Gamma$ we make two additional assumptions regarding the shape of $\Omega$; namely, that the curvatures $\kappa_{1}, \kappa_{2}$, and $K$ are constrained in the following way:

(a) There exist constants $g$ and $G$ such that

$$
0<g \leq K(x) \leq G \quad \forall x \in \Gamma .
$$

(b) There exists a constant $H$ such that

$$
0 \leq \kappa_{1}^{2}+\kappa_{2}^{2} \leq H^{2} \quad \text { on } \Gamma \text {. }
$$

Note that these assumptions allow cases where $\kappa_{1}$ and $\kappa_{2}$ can be of opposite signs.

The Frenet-Serret [34-36] formulae in this case, providing that there is no torsion, are then

$$
\begin{array}{ll}
\frac{\partial \mathbf{n}}{\partial s_{1}}=-\kappa_{1} \boldsymbol{\tau}_{1} & \frac{\partial \mathbf{n}}{\partial s_{2}}=-\kappa_{2} \boldsymbol{\tau}_{2} \\
\frac{\partial \boldsymbol{\tau}_{1}}{\partial s_{1}}=\kappa_{1} \mathbf{n} & \frac{\partial \boldsymbol{\tau}_{1}}{\partial s_{2}}=0 \\
\frac{\partial \boldsymbol{\tau}_{2}}{\partial s_{1}}=0 & \frac{\partial \boldsymbol{\tau}_{2}}{\partial s_{2}}=\kappa_{2} \mathbf{n} .
\end{array}
$$

The surface gradient $\nabla_{s}$ of a scalar function $f$ may be written as

$$
\gamma_{o}(\nabla f)=\nabla_{s}\left(\gamma_{o} f\right)+\mathbf{n} \gamma_{1} f
$$

where the trace operator $\gamma_{1}$ denotes the normal derivative. Also consider

$$
\begin{gathered}
\nabla_{s} f=\frac{\partial f}{\partial s_{1}} \boldsymbol{\tau}_{1}+\frac{\partial f}{\partial s_{2}} \boldsymbol{\tau}_{2}, \\
\Delta_{s} f=\nabla_{s} \cdot\left(\nabla_{s} f\right)=\frac{\partial^{2} f}{\partial^{2} s_{1}^{2}}+\frac{\partial^{2} f}{\partial^{2} s_{2}^{2}} .
\end{gathered}
$$

If $\mathbf{f}$ is a vector field defined on $\Gamma$, the surface gradient $\nabla_{s}$ is defined as the tensor

$$
\nabla_{s} \mathbf{f}=\frac{\partial \mathbf{f}}{\partial s_{1}} \otimes \tau_{1}+\frac{\partial \mathbf{f}}{\partial s_{2}} \otimes \boldsymbol{\tau}_{2} .
$$

Surface divergence and surface curl are defined as

$$
\begin{gathered}
\nabla_{s} \cdot \mathbf{f}=\boldsymbol{\tau}_{\mathbf{1}} \cdot \frac{\partial \mathbf{f}}{\partial s_{1}}+\boldsymbol{\tau}_{\mathbf{2}} \cdot \frac{\partial \mathbf{f}}{\partial s_{2}} \\
\nabla_{s} \wedge \mathbf{f}=\boldsymbol{\tau}_{\mathbf{1}} \wedge \frac{\partial \mathbf{f}}{\partial s_{1}}+\tau_{2} \wedge \frac{\partial \mathbf{f}}{\partial s_{2}}
\end{gathered}
$$

The relationship between the surface operators and the volume operators for a function defined in $\Omega$ is given by

$$
\begin{gathered}
\gamma_{o}(\nabla \mathbf{f})=\nabla_{s} \gamma_{o} \mathbf{f}+\gamma_{o}[(\mathbf{n} \cdot \nabla) \mathbf{f}] \otimes \mathbf{n}, \\
\gamma_{o}(\nabla \cdot \mathbf{f})=\nabla_{s} \cdot \gamma_{o} \mathbf{f}+\gamma_{o}[(\mathbf{n} \cdot \nabla) \mathbf{f}] \cdot \mathbf{n}, \\
\gamma_{o}(\nabla \wedge \mathbf{f})=\nabla_{s} \wedge \gamma_{o} \mathbf{f}+\mathbf{n} \wedge \gamma_{o}[(\mathbf{n} \cdot \nabla) \mathbf{f}] .
\end{gathered}
$$

We use (20)-(25) to prove more important results to make the calculations easier.

Lemma 2. Let $\boldsymbol{\tau}_{1}$ and $\boldsymbol{\tau}_{2}$ be two orthogonal unit tangential vectors and let $\mathbf{n}$ be the exterior unit normal vector to $\Gamma$. Let $\alpha$, $\beta$, and $\gamma$ be scalar functions; then

$$
\begin{aligned}
& \text { (a) } \nabla_{s} \cdot\left(\alpha \boldsymbol{\tau}_{1}\right)=\frac{\partial \alpha}{\partial s_{1}} \\
& \text { (b) } \nabla_{s} \cdot\left(\beta \boldsymbol{\tau}_{2}\right)=\frac{\partial \beta}{\partial s_{2}} \\
& \text { (c) } \nabla_{s} \cdot(\gamma \mathbf{n})=-\gamma K .
\end{aligned}
$$

Proof. Consider the following:

$$
\begin{aligned}
& \text { (a) } \nabla_{s} \cdot\left(\alpha \boldsymbol{\tau}_{1}\right)=\boldsymbol{\tau}_{1} \cdot \frac{\partial}{\partial s_{1}}\left(\alpha \boldsymbol{\tau}_{1}\right)+\boldsymbol{\tau}_{2} \cdot \frac{\partial}{\partial s_{2}}\left(\alpha \boldsymbol{\tau}_{1}\right)=\frac{\partial \alpha}{\partial s_{1}} \text {. } \\
& \text { (b) Similar to (a). } \\
& \text { (c) } \nabla_{s} \cdot(\gamma \mathbf{n})=\boldsymbol{\tau}_{1} \cdot\left[\frac{\partial \gamma}{\partial s_{1}} \mathbf{n}-\kappa_{1} \gamma \boldsymbol{\tau}_{1}\right] \\
& +\boldsymbol{\tau}_{2} \cdot\left[\frac{\partial \gamma}{\partial s_{2}} \mathbf{n}-\gamma \kappa_{2} \boldsymbol{\tau}_{2}\right] \\
& =-\gamma\left[\kappa_{1}+\kappa_{2}\right]=-\gamma K \text {. }
\end{aligned}
$$

We shall apply the expressions above to v. By the FrenetSerret formulae (torsion is zero) $\left(\partial / \partial s_{1}\right)\left(\gamma_{o} \mathbf{v}\right)=-\left(\partial \eta / \partial s_{1}\right) \mathbf{n}-$ $\eta\left(\partial \mathbf{n} / \partial s_{1}\right)=-\left(\partial \eta / \partial s_{1}\right) \mathbf{n}+\kappa_{1} \tau_{1} \eta$, and, similarly, $\partial\left(\gamma_{0} \mathbf{v}\right) / \partial s_{2}=$ $-\left(\partial \eta / \partial s_{2}\right) \mathbf{n}+\kappa_{2} \boldsymbol{\tau}_{2} \eta$. Hence 


$$
\begin{aligned}
& \nabla_{s} \gamma_{o} \mathbf{v}=\eta\left[\kappa_{1} \tau_{1} \otimes \tau_{1}+\kappa_{2} \tau_{2} \otimes \tau_{2}\right] \\
& -\left[\frac{\partial \eta}{\partial s_{1}} \mathbf{n} \otimes \boldsymbol{\tau}_{\mathbf{1}}+\frac{\partial \eta}{\partial s_{2}} \mathbf{n} \otimes \boldsymbol{\tau}_{\mathbf{2}}\right] \\
& =\eta\left[\kappa_{1} \tau_{1} \otimes \tau_{1}+\kappa_{2} \tau_{2} \otimes \tau_{2}\right] \\
& -\mathbf{n} \otimes\left[\frac{\partial \eta}{\partial s_{1}} \boldsymbol{\tau}_{1}+\frac{\partial \eta}{\partial s_{2}} \boldsymbol{\tau}_{\mathbf{2}}\right] \\
& =\eta\left[\kappa_{1} \boldsymbol{\tau}_{1} \otimes \boldsymbol{\tau}_{1}+\kappa_{2} \boldsymbol{\tau}_{2} \otimes \boldsymbol{\tau}_{2}\right]-\mathbf{n} \otimes \nabla_{s} \eta .
\end{aligned}
$$

The transpose is given by

$$
\left(\nabla_{s}\left(\gamma_{o} \mathbf{v}\right)\right)^{T}=\eta\left[\kappa_{1} \boldsymbol{\tau}_{\mathbf{1}} \otimes \boldsymbol{\tau}_{\mathbf{1}}+\kappa_{2} \boldsymbol{\tau}_{\mathbf{2}} \otimes \boldsymbol{\tau}_{\mathbf{2}}\right]-\nabla_{s} \eta \otimes \mathbf{n}
$$

To find an expression for $\mathbf{A}$ at $\Gamma$, we need an expression for $\nabla \mathbf{v}$ on the boundary:

$$
\gamma_{o}(\nabla \mathbf{v})=\nabla_{s}\left(\gamma_{o} \mathbf{v}\right)+\gamma_{o}[(\mathbf{n} \cdot \nabla) \mathbf{v}] \otimes \mathbf{n}
$$

Although we know that the divergence of $\mathbf{v}$ will be zero, it is helpful to observe that $\theta=\gamma_{o}(\nabla \cdot \mathbf{v})=\nabla_{s} \cdot\left(\gamma_{o} \mathbf{v}\right)+\gamma_{o}[(\mathbf{n} \cdot \nabla) \mathbf{v}] \cdot \mathbf{n}$, where

$$
\begin{aligned}
\nabla_{s} \cdot\left(\gamma_{o} \mathbf{v}\right) & =-\boldsymbol{\tau}_{\mathbf{1}} \cdot \frac{\partial}{\partial s_{1}}(\eta \mathbf{n})-\boldsymbol{\tau}_{\mathbf{2}} \cdot \frac{\partial}{\partial s_{2}}(\eta \mathbf{n}) \\
& =-\boldsymbol{\tau}_{\mathbf{1}} \cdot\left[\frac{\partial \eta}{\partial s_{1}} \mathbf{n}-\eta \kappa_{1} \boldsymbol{\tau}_{\mathbf{1}}\right]-\boldsymbol{\tau}_{\mathbf{2}} \cdot\left(\frac{\partial \eta}{\partial s_{2}} \mathbf{n}-\eta \kappa_{2} \boldsymbol{\tau}_{\mathbf{2}}\right) \\
& =\eta\left(\kappa_{1}+\kappa_{2}\right) \\
& =\eta K .
\end{aligned}
$$

Hence $\theta=\eta K+\gamma_{o}[(\mathbf{n} \cdot \nabla) \mathbf{v}] \cdot \mathbf{n}$.

We proceed to find expressions for $\gamma_{o}(\mathbf{v} \cdot \nabla) \mathbf{v}, \gamma_{o}(\nabla \mathbf{v})$, and $\gamma_{o}(\nabla \mathbf{v})^{T}$.

We know that $\boldsymbol{\omega} \wedge \mathbf{n}=\mathbf{W}(\mathbf{v}) \mathbf{n}=(\mathbf{n} \cdot \nabla) \mathbf{v}-(\nabla \mathbf{v})^{T} \mathbf{n}$, and

$$
\begin{aligned}
\gamma_{o}(\nabla \mathbf{v})^{T} \mathbf{n} & =\left(\nabla_{s}\left(\gamma_{o} \mathbf{v}\right)\right)^{T} \mathbf{n}+\gamma_{o}([\mathbf{n} \cdot(\mathbf{n} \cdot \nabla) \mathbf{v}] \mathbf{n}) \\
& =-\left(\nabla_{s} \eta\right)+(\theta-K \eta) \mathbf{n} .
\end{aligned}
$$

Therefore,

$$
(\mathbf{n} \cdot \nabla) \gamma_{o} \mathbf{v}=\left(\gamma_{\mathbf{o}} \boldsymbol{\omega} \wedge \mathbf{n}\right)-\nabla_{s} \eta+(\theta-K \eta) \mathbf{n} .
$$

Multiply (34) with $-\eta$ to obtain

$$
\gamma_{o}(\mathbf{v} \cdot \nabla) \mathbf{v}=-\eta(\mathbf{n} \cdot \nabla) \gamma_{o} \mathbf{v}=K \eta^{2} \mathbf{n}+\eta\left[\nabla_{s} \eta-\gamma_{0} \boldsymbol{\omega} \wedge \mathbf{n}\right]
$$

From (31) we now obtain

$$
\begin{aligned}
\gamma_{o}(\nabla \mathbf{v})= & \nabla_{s} \mathbf{v}+\left[\boldsymbol{\gamma}_{\mathbf{o}} \boldsymbol{\omega} \wedge \mathbf{n}-\nabla_{s} \eta+(\theta-K \eta) \mathbf{n}\right] \otimes \mathbf{n} \\
= & \eta\left[\kappa_{1} \boldsymbol{\tau}_{\mathbf{1}} \otimes \boldsymbol{\tau}_{\mathbf{1}}+\kappa_{2} \boldsymbol{\tau}_{\mathbf{2}} \otimes \boldsymbol{\tau}_{\mathbf{2}}\right] \\
& -\mathbf{n} \otimes \nabla_{s} \eta-\nabla_{s} \eta \otimes \mathbf{n}+\left(\gamma_{\mathbf{o}} \boldsymbol{\omega} \wedge \mathbf{n}\right) \otimes \mathbf{n} \\
& +(\theta-K \eta) \mathbf{n} \otimes \mathbf{n} \\
= & \eta\left[\kappa_{1} \boldsymbol{\tau}_{\mathbf{1}} \otimes \boldsymbol{\tau}_{\mathbf{1}}+\kappa_{2} \boldsymbol{\tau}_{\mathbf{2}} \otimes \boldsymbol{\tau}_{\mathbf{2}}-K \mathbf{n} \otimes \mathbf{n}\right] \\
& -\left[\mathbf{n} \otimes \nabla_{s} \eta+\nabla_{s} \eta \otimes \mathbf{n}\right] \\
& +\left(\gamma_{\mathbf{o}} \boldsymbol{\omega} \wedge \mathbf{n}\right) \otimes \mathbf{n}+\theta \mathbf{n} \otimes \mathbf{n} .
\end{aligned}
$$

The transpose is

$$
\begin{aligned}
\gamma_{o}(\nabla \mathbf{v})^{T}= & \eta\left[\kappa_{1} \boldsymbol{\tau}_{\mathbf{1}} \otimes \boldsymbol{\tau}_{\mathbf{1}}+\kappa_{2} \boldsymbol{\tau}_{\mathbf{2}} \otimes \boldsymbol{\tau}_{\mathbf{2}}-K \mathbf{n} \otimes \mathbf{n}\right] \\
& -\left[\mathbf{n} \otimes \nabla_{s} \eta+\nabla_{s} \eta \otimes \mathbf{n}\right] \\
& +\mathbf{n} \otimes\left(\boldsymbol{\gamma}_{\mathbf{o}} \boldsymbol{\omega} \wedge \mathbf{n}\right)+\theta \mathbf{n} \otimes \mathbf{n} .
\end{aligned}
$$

Thus we have

$$
\begin{aligned}
\gamma_{o}(\mathbf{A})= & \gamma_{o}(\nabla \mathbf{v})+\gamma_{o}(\nabla \mathbf{v})^{T} \\
= & \nabla_{s} \gamma_{o} \mathbf{v}+\left(\nabla_{s} \gamma_{o} \mathbf{v}\right)^{T}+\gamma_{o}([(\mathbf{n} \cdot \nabla) \mathbf{v}] \otimes \mathbf{n}) \\
& +\gamma_{o}(\mathbf{n} \otimes[(\mathbf{n} \cdot \nabla) \mathbf{v}]) \\
= & 2 \eta\left[\kappa_{1} \boldsymbol{\tau}_{\mathbf{1}} \otimes \boldsymbol{\tau}_{\mathbf{1}}+\kappa_{2} \boldsymbol{\tau}_{\mathbf{2}} \otimes \boldsymbol{\tau}_{\mathbf{2}}-K \mathbf{n} \otimes \mathbf{n}\right] \\
& -2\left[\mathbf{n} \otimes \nabla_{s} \eta+\nabla_{s} \eta \otimes \mathbf{n}\right] \\
& +\mathbf{n} \otimes\left(\gamma_{\mathbf{o}} \boldsymbol{\omega} \wedge \mathbf{n}\right)+\left(\gamma_{\mathbf{o}} \boldsymbol{\omega} \wedge \mathbf{n}\right) \otimes \mathbf{n} .+2 \theta \mathbf{n} \otimes \mathbf{n} .
\end{aligned}
$$

Let us define the symmetrical tensors $\mathbf{M}$ and $\mathbf{N}$ by

$$
\begin{aligned}
\mathbf{M}= & {\left[K \mathbf{n} \otimes \mathbf{n}-\left(\kappa_{1} \boldsymbol{\tau}_{\mathbf{1}} \otimes \boldsymbol{\tau}_{\mathbf{1}}+\kappa_{2} \boldsymbol{\tau}_{\mathbf{2}} \otimes \boldsymbol{\tau}_{\mathbf{2}}\right)\right], } \\
\mathbf{N}= & \mathbf{n} \otimes\left(\gamma_{\mathbf{o}} \boldsymbol{\omega} \wedge \mathbf{n}\right)+\left(\boldsymbol{\gamma}_{\mathbf{0}} \boldsymbol{\omega} \wedge \mathbf{n}\right) \otimes \mathbf{n} \\
& +2 \theta \mathbf{n} \otimes \mathbf{n}-2\left(\mathbf{n} \otimes \nabla_{s} \eta+\nabla_{s} \eta \otimes \mathbf{n}\right) \\
= & \mathbf{n} \otimes\left[\gamma_{\mathbf{o}} \boldsymbol{\omega} \wedge \mathbf{n}-2 \nabla_{s} \eta\right] \\
& +\left[\boldsymbol{\gamma}_{\mathbf{o}} \boldsymbol{\omega} \wedge \mathbf{n}-2 \nabla_{s} \eta\right] \otimes \mathbf{n}+2 \theta \mathbf{n} \otimes \mathbf{n} \\
= & \mathbf{n} \otimes \psi+\psi \otimes \mathbf{n}-2 \theta \mathbf{n} \otimes \mathbf{n},
\end{aligned}
$$

with

$$
\psi=\gamma_{\mathbf{o}} \boldsymbol{\omega} \wedge \mathbf{n}-2 \nabla_{s} \eta
$$

a tangential vector. Then, for a vector field of the form $\mathbf{v}=$ $-\eta \mathbf{n}$ on $\Gamma$, from (38) we have

$$
\gamma_{o} \mathbf{A}=-2 \eta \mathbf{M}+\mathbf{N} \text { on } \Gamma
$$


In local coordinates we have the representations

$$
\begin{gathered}
\mathbf{M}=\left(\begin{array}{ccc}
-\kappa_{1} & 0 & 0 \\
0 & -\kappa_{2} & 0 \\
0 & 0 & K
\end{array}\right), \\
\mathbf{N}=\left(\begin{array}{ccc}
0 & 0 & \psi \cdot \tau_{1} \\
0 & 0 & \psi \cdot \tau_{2} \\
\psi \cdot \tau_{1} & \psi \cdot \tau_{2} & -2 \theta
\end{array}\right) .
\end{gathered}
$$

If $\nabla \cdot \mathbf{v}=0$, it follows that $\operatorname{tr} \mathbf{A}=0$, which is in line with incompressibility.

We would further like to obtain expressions for the terms $\mathbf{n} \cdot \Delta \mathbf{v}, \mathbf{n} \cdot[(\mathbf{v} \cdot \nabla) \mathbf{A}] \mathbf{n}$, and $\mathbf{n} \cdot[\mathbf{A W}-\mathbf{W A}] \mathbf{n}$ on the boundary $\Gamma$.

Lemma 3. Let $\mathbf{n}$ be the exterior normal to the boundary $\Gamma, \mathbf{v} \in$ $\mathscr{D}$, and $\mathbf{A}=-2 \eta \mathbf{M}+\mathbf{N}$ with $\mathbf{M}$ and $\mathbf{N}$ as defined in (39). We assume that $\nabla \cdot \mathbf{v}=0$ and $\gamma_{\mathbf{o}} \boldsymbol{\omega} \wedge \mathbf{n}=2 \nabla_{s} \eta$, which implies that $\mathbf{N}=0$. Then

$$
\begin{aligned}
& \text { (a) } \gamma_{o}(-\mathbf{n} \cdot \Delta \mathbf{v})=\Delta_{s} \eta \\
& \text { (b) } \gamma_{o}[\mathbf{n} \cdot[(\mathbf{v} \cdot \nabla) \mathbf{A}] \mathbf{n}]=-4 \eta^{2} K_{G}-2 \eta \Delta_{s} \eta \\
& \text { (c) } \gamma_{o}[\mathbf{n} \cdot[\mathbf{A W}-\mathbf{W A}] \mathbf{n}]=0,
\end{aligned}
$$

where $K_{G}$ denotes the Gauss-curvature.

Proof. (a) We have chosen $\boldsymbol{\tau}_{1}, \boldsymbol{\tau}_{2}$, and $\mathbf{n}$ so that $\boldsymbol{\tau}_{1} \wedge \boldsymbol{\tau}_{\mathbf{2}}=\mathbf{n}$. In view of the incompressibility and the fact that there is zero tangential velocity

$$
\begin{aligned}
\gamma_{o}(-\Delta \mathbf{v})= & \nabla \wedge \gamma_{\mathbf{o}} \boldsymbol{\omega} \\
= & \boldsymbol{\tau}_{\mathbf{1}} \wedge \partial_{s_{1}}\left[\eta_{1} \boldsymbol{\tau}_{2}-\eta_{2} \boldsymbol{\tau}_{1}\right]+\boldsymbol{\tau}_{2} \wedge \partial_{s_{2}}\left[\eta_{1} \boldsymbol{\tau}_{2}-\eta_{2} \boldsymbol{\tau}_{\mathbf{1}}\right] \\
& + \text { a tangential term } \\
= & \boldsymbol{\tau}_{\mathbf{1}} \wedge\left[\partial_{s_{1}} \eta_{1} \boldsymbol{\tau}_{2}-\eta_{2} \kappa_{1} \mathbf{n}\right] \\
& +\boldsymbol{\tau}_{\mathbf{2}} \wedge\left[\eta_{1} \kappa_{2} \mathbf{n}-\partial_{s_{1}} \eta_{2} \boldsymbol{\tau}_{1}\right]+\cdots \\
= & \left(\partial_{s_{1}} \eta_{1}+\partial_{s_{2}} \eta_{2}\right) \mathbf{n}+\cdots \\
= & \Delta_{s} \eta \mathbf{n} .
\end{aligned}
$$

(b) Consider the tensor $\nabla \cdot \mathbf{A}$ built from "row vectors" with $\left(\mathbf{e}_{1}, \mathbf{e}_{2}, \mathbf{e}_{3}\right)$ a basis for $\mathbf{R}^{3}$. Then

$$
\begin{aligned}
\nabla \cdot \mathbf{A} & =\left(\begin{array}{l}
\nabla \cdot \mathbf{A e}_{1} \\
\nabla \cdot \mathbf{A e}_{2} \\
\nabla \cdot \mathbf{A e}_{3}
\end{array}\right) \\
& =\left(\begin{array}{c}
\nabla_{s} \cdot \mathbf{A e}_{1} \\
\nabla_{s} \cdot \mathbf{A e}_{2} \\
\nabla_{s} \cdot \mathbf{A e}_{3}
\end{array}\right)+\left(\begin{array}{l}
\mathbf{n} \cdot\left[(\mathbf{n} \cdot \nabla) \mathbf{A e}_{1}\right] \\
\mathbf{n} \cdot\left[(\mathbf{n} \cdot \nabla) \mathbf{A e}_{2}\right] \\
\mathbf{n} \cdot\left[(\mathbf{n} \cdot \nabla) \mathbf{A e}_{3}\right]
\end{array}\right) \\
& =\nabla_{s} \cdot \mathbf{A}+[(\mathbf{n} \cdot \nabla) \mathbf{A}] \mathbf{n} .
\end{aligned}
$$

Hence, $\gamma_{o}[(\mathbf{n} \cdot \nabla) \mathbf{A}] \mathbf{n}=\gamma_{o}[\nabla \cdot \mathbf{A}]-\nabla_{s} \cdot \gamma_{o}(\mathbf{A})$.
Furthermore,

$$
\nabla_{s} \cdot \gamma_{o}[\mathbf{A}]=-2\left(M \nabla_{s} \eta+\eta \nabla_{s} \cdot M\right)
$$

and now

$$
\begin{aligned}
\mathbf{n} \cdot\left[\nabla_{s} \cdot\left[\gamma_{o} \mathbf{A}\right]\right] & =-2\left(\mathbf{n} \cdot M \nabla_{s} \eta+\eta \mathbf{n} \cdot\left(\nabla_{s} \cdot M\right)\right) \\
& =-2 \eta \mathbf{n} \cdot\left(\nabla_{s} \cdot M\right) .
\end{aligned}
$$

Here we used the fact that $M \mathbf{n}=-K \mathbf{n}$. Determine $\mathbf{n} \cdot\left[\nabla_{s} \cdot M\right]$ term by term to obtain

$$
\begin{aligned}
\mathbf{n} \cdot\left[\nabla_{s} \cdot\left[\gamma_{o} \mathbf{A}\right]\right] & =-2 \eta\left(\kappa_{1}^{2}+\kappa_{2}^{2}-K^{2}\right) \\
& =-2 \eta\left(2 \kappa_{1} \kappa_{2}\right)=-4 K_{G} \eta .
\end{aligned}
$$

$K_{G}$ denotes the Gauss-curvature and is bounded by assumptions (17) and (18). Hence

$$
\gamma_{o} \mathbf{n} \cdot[(\mathbf{n} \cdot \nabla) \mathbf{A}] \mathbf{n}=\gamma_{o}[\mathbf{n} \cdot \Delta \mathbf{v}]-4 \eta K_{G}=-4 \eta K_{G}-\Delta_{s} \eta .
$$

The term we use in the proof of (57) is therefore

$$
-\eta \gamma_{o}[\mathbf{n} \cdot[(\mathbf{n} \cdot \nabla) \mathbf{A}] \mathbf{n}]=+4 \eta^{2} K_{G}+\eta \Delta_{s} \eta
$$

(c) $\mathbf{n} \cdot(\mathbf{A W}-\mathbf{W A}) \mathbf{n}=\mathbf{A n} \cdot \mathbf{W n}+\mathbf{W n} \cdot \mathbf{A n}=2 \mathbf{A n} \cdot \mathbf{W n}$.

Here we make use of the additional boundary conditions (52) and (55) and the fact that $\mathbf{W n}=\gamma_{\mathbf{o}} \boldsymbol{\omega} \wedge \mathbf{n}$ to obtain that

$$
\begin{aligned}
\mathbf{A n} \cdot \mathbf{W n} & =\mathbf{A n} \cdot\left(\gamma_{\mathbf{o}} \boldsymbol{\omega} \wedge \mathbf{n}\right) \\
& =\left(\gamma_{\mathbf{o}} \boldsymbol{\omega} \wedge \mathbf{n}\right) \cdot[-2 \eta K \mathbf{n}] \\
& =0 .
\end{aligned}
$$

6.1. Explicit Form of the Dynamic Boundary Condition. It is shown that for a smooth two-dimensional manifold $\Gamma$ contained in a domain $\Omega \subset \mathbf{R}^{3}$ the following is true for a vector field $\mathbf{v}$ which is of the form $\mathbf{v}=-\eta \mathbf{n}$ on $\Gamma$ :

$$
\gamma_{o}[\mathbf{A}]=-2 \eta \mathbf{M}+\mathbf{N}
$$

where $\mathbf{M}$ and $\mathbf{N}$ are defined in (39).

If $\mathbf{v}$ is solenoidal, as in the case under consideration, $\theta=$ 0. A straightforward application of Stokes' theorem shows that $\boldsymbol{\omega}$ is tangential to the boundary, which implies that $\psi$ is tangential to the boundary. Indeed, let $\Gamma^{\prime}$ be any patch of the surface $\Gamma$; then

$$
\int_{\Gamma^{\prime}}(\nabla \wedge \mathbf{f}) \cdot \mathbf{n} d s=\int_{\partial \Gamma^{\prime}} \mathbf{f} \cdot d \boldsymbol{\tau}
$$

where $d \boldsymbol{\tau}$ is a vector tangential to the boundary. Now if $\mathbf{f}=$ $\gamma_{o} \mathbf{v}=-\eta \mathbf{n}$, then $\int_{\partial \Gamma^{\prime}} \mathbf{f} \cdot d \boldsymbol{\tau}=0$, and that implies that $\int_{\Gamma^{\prime}}(\nabla \wedge \mathbf{v}) \cdot$ $\mathbf{n} d s=0$ for all $\Gamma^{\prime} \subset \Gamma$, which in turn implies that $(\nabla \wedge \mathbf{v}) \cdot \mathbf{n}=0$. 
In the problem under consideration we shall assume that the "rate of deformation" tensor A has precisely the form (52) on the boundary $\Gamma$ with $\mathbf{n}$ the unit exterior normal (the traditional rate of deformation is defined as $\mathbf{D}=(1 / 2) \mathbf{A})$.

We shall consider a kinematic boundary condition, which has a physical meaning in that there are no tangential components of deformation at the interface boundary. This concerns the form of the tensor $\mathbf{N}$.

Towards this, we observe from (52) that

$$
\gamma_{0}[\mathbf{A n}]=-2 K \eta \mathbf{n}+\psi
$$

It follows from (54) that there are no tangential components of deformation at $\Gamma$ if and only if $\psi=0$; that is,

$$
\gamma_{\mathbf{o}} \boldsymbol{\omega} \wedge \mathbf{n}=2 \nabla_{s} \eta
$$

This is the kinematic boundary condition.

The various terms in $\mathbf{n} \cdot \mathbf{T n}$, with $\mathbf{T}$ on a surface $\Gamma$, given by (8), may be expressed as follows (see Lemma 3):

$$
\begin{gathered}
\mathbf{n} \cdot \gamma_{o} \mathbf{A} \mathbf{n}=-2 K \eta \\
\mathbf{n} \cdot \gamma_{o} \partial_{t}[\mathbf{A n}]=\partial_{t}[\mathbf{n} \cdot \mathbf{A} \mathbf{n}]=-2 K \eta_{t} \\
\gamma_{o}[\mathbf{n} \cdot[(\mathbf{v} \cdot \nabla) \mathbf{A}] \mathbf{n}]=+4 K_{G} \eta^{2}+\eta \Delta_{s} \eta \\
\mathbf{n} \cdot[\mathbf{A W}-\mathbf{W A}] \mathbf{n}=0 .
\end{gathered}
$$
$\Gamma$

Guided by these expressions and (8), we assume that, at

$$
\mathbf{n} \cdot \gamma_{o} \mathbf{T n}=-\left[\gamma_{o} p+2 \mu K \eta+2 \alpha K \eta_{t}-4 \alpha K_{G} \eta^{2}-\alpha \eta \Delta_{s} \eta\right] .
$$

For the stress tensor $\mathbf{T}^{\prime}$ in the fluid exterior to $\Omega$ we assume that $\mathbf{n} \cdot \mathbf{T}^{\prime} \mathbf{n}=\ell(t)$. This amounts to the situation where the fluid in $\Omega_{o}$ is at rest. As a result we have

$$
\begin{aligned}
\mathbf{n} \cdot(\delta \mathbf{T}) \mathbf{n}= & \mathbf{n} \cdot\left[\mathbf{T}-\mathbf{T}^{\prime}\right] \mathbf{n} \\
= & -\left[\gamma_{o} p+2 \mu K \eta+2 \alpha K \eta_{t}-4 \alpha K_{G} \eta^{2}-\alpha \eta \Delta_{s} \eta\right] \\
& -\ell(t) .
\end{aligned}
$$

From (13), (14), and (60) we obtain

$$
\sigma^{-1 / 2}(\sigma+2 \alpha K) \eta_{t}+\sigma^{-1 / 2} \gamma_{0} p=s(\eta)
$$

with $s(\eta)=\sigma^{-1 / 2}\left[\left(-k+4 \alpha K_{G}\right) \eta^{2}-2 \mu \eta K+\alpha \eta \Delta_{s} \eta-\ell(t)\right]$, and $k=\zeta \rho$.

Equation (61) is the explicit form of the dynamic boundary condition.

\section{An Alternative Model: Problem $\mathscr{A}$}

Although it was possible to prove stability and uniqueness for the original model (see $[33,37]$ ), we could not find a way to a possible proof of existence for a classical solution. In this chapter we describe an alternative model which displays all the properties of the original problem with respect to stability and uniqueness.

In the alternative model the dynamics at the boundary are formulated by assuming a "shear flow" of the form

$$
\mathbf{v}^{*}(y, t)=-\eta\left(s_{1}, s_{2}, t\right) \mathbf{n}(y)
$$

with $s_{1}$ and $s_{2}$ as the surface parameters (like arc length). It is assumed that the "body force" acting on the shearing fluid at the boundary is proportional to the difference between the pressures $\gamma_{o} p$ and $\ell(t)$. Under these assumptions the equation governing the evolution of $\eta$ is

$$
\partial_{t}\left[\rho \eta-\alpha \Delta_{s} \eta\right]+\delta^{-1} \gamma_{o} p=\mu \Delta_{s} \eta+\delta^{-1} \ell(t),
$$

where $\gamma_{o} \mathbf{v}=-\eta \mathbf{n}$, and $p$ is the resulting pressure through the boundary with thickness $\delta . \Delta_{s}$ is the Laplace-Beltrami operator $\left(\Delta_{s}=\nabla_{s} \cdot \nabla_{s}\right)$ and $\nabla_{s}$ denotes the surface gradient. The parameter $\delta$ has the physical dimension of length and may be thought of as the "thickness" of the "shear layer" (see [38], Sect 123, p. 506). Equation (63) is derived by calculating the stress tensor for a shear flow and noticing that terms of the form $\mathbf{v}^{*} \cdot \nabla_{s}$ vanish. The term $\delta^{-1} \ell(t)$ may be left out since, as before, it disappears when projections are taken. The kinematic boundary condition is still imposed.

7.1. Definitions. All spaces of vector fields are denoted by boldface letters.

(1) Let $\Omega$ be a bounded domain in $\mathbf{R}^{3}$ with a smooth boundary $\Gamma$ (of class $C^{\infty}$ ), $\Omega_{T}=\Omega \times(0, T)$, and $\Gamma_{T}=\Gamma \times(0, T)$.

(2) $H^{m, q}(\Omega)$, for $m$ a nonnegative integer and $1<q<\infty$, is the usual Sobolev space (of real-valued functions) embedded in $L^{q}(\Omega)$ with norm $\|\cdot\|_{m, q} . H^{m}(\Omega)$, for $m$ a nonnegative integer, denotes the Sobolev space $H^{m, 2}(\Omega)$ of order $m$. By this agreement $\mathbf{H}^{m}(\Omega)$ is the Sobolev space of three vector fields and the components are elements of $H^{m}(\Omega)$. In particular, the norm and scalar products in $\mathbf{H}^{\mathbf{l}}(\Omega)$ are defined by $\|\mathbf{u}\|_{1}^{2}=\|\mathbf{u}\|_{\Omega}^{2}+\|\nabla \mathbf{u}\|_{\Omega}^{2}$ and $(\mathbf{u}, \mathbf{v})_{1}=\int_{\Omega} \mathbf{u} \cdot \mathbf{v} d x+\int_{\Omega} \nabla \mathbf{u}$ : $\nabla \mathbf{v} d x$.

(3) With the above notation $\mathbf{H}^{0}(\Omega)$ denotes the Hilbert space $\mathbf{L}^{2}(\Omega)$ of vector functions $\mathbf{u}(x)=\left(u_{1}(x)\right.$, $\left.u_{2}(x), u_{3}(x)\right)$, with $x \in \Omega$, for which $|\mathbf{u}|^{2}$ is integrable on $\Omega$. The norm and scalar products for $\mathbf{u}, \mathbf{v} \in \mathbf{L}^{2}(\Omega)$ are defined as $\|\mathbf{u}\|_{\Omega}^{2}=\int_{\Omega}|\mathbf{u}|^{2} d x$ and $(\mathbf{u}, \mathbf{v})_{\Omega}=\int_{\Omega} \mathbf{u}$. $\mathbf{v} d x$.

(4) There exists a linear continuous operator $\gamma_{o} \in \mathscr{L}\left(\mathbf{H}^{1}\right.$ $\left.(\Omega), \mathbf{L}^{2}(\partial \Omega)\right)$, called the trace operator, such that $\gamma_{o} \mathbf{u}$ $=$ the "restriction" of $\mathbf{u}$ to $\partial \Omega$ for every function $\mathbf{u} \epsilon$ $\mathbf{H}^{1}(\Omega)$ which is continuous in $\bar{\Omega}$. The space $\mathbf{H}_{o}^{1}(\Omega)$ is the kernel of $\gamma_{o}$. The image space $\gamma_{o}\left(\mathbf{H}^{1}(\Omega)\right)$ is a dense subspace of $\mathbf{L}^{2}(\Gamma)$ denoted by $\mathbf{H}^{1 / 2}(\Gamma)$. The trace operator is bounded. Indeed, there exists a constant $C_{1}>0$ such that

$$
\left\|\gamma_{o} \mathbf{u}\right\|_{\Gamma} \leq C_{1}\|\mathbf{u}\|_{1} \quad \forall \mathbf{u} \in \mathbf{H}^{1}(\Omega) .
$$


Reference [39, Theorem 9.4, page 41]. We shall refer to this result (64) as the Trace theorem.

(5) For the deformation we use the following notation for the norm and scalar products: $(\mathbf{A}(\mathbf{u}), \mathbf{A}(\mathbf{v}))_{\Omega}=$ $\int_{\Omega} \mathbf{A}(\mathbf{u}): \mathbf{A}(\mathbf{v}) d x$ and $\|\mathbf{A}\|_{\Omega}^{2}=\int_{\Omega}|\mathbf{A}|^{2} d x$.

(6) We define the domain $\mathscr{D}$ by

$$
\begin{aligned}
\mathscr{D}=\{\mathbf{v} & \in \mathbf{H}^{2}(\Omega): \nabla \cdot \mathbf{v}=0 \text { in } \Omega, \gamma_{o} \mathbf{v} \\
& \left.=-\eta \mathbf{n} \in \mathbf{L}^{2}(\Gamma), \mathbf{A}(\mathbf{v})=-2 \eta \mathbf{M} \text { on } \Gamma\right\} .
\end{aligned}
$$

$\mathscr{D}$ is a closed subspace of $\mathbf{H}^{2}(\Omega)$. Elements of $\mathscr{D}$ satisfy the kinematical boundary conditions (55).

(7) $\mathbf{H}_{\varsigma}^{1}(\Omega)$ denotes the closure of $\mathscr{D}$ in $\mathbf{H}^{1}(\Omega)$ with respect to the Sobolev norm $\|\cdot\|_{1}$.

(8) The norm of $\gamma_{o} \mathbf{v} \in \mathbf{L}^{2}(\Gamma)$ on the boundary $\Gamma$ is chosen as

$$
\left\|\gamma_{o} \mathbf{v}\right\|_{\Gamma}^{2}=\|\eta\|_{\Gamma}^{2}=\int_{\Gamma} \sigma(x)\left|\gamma_{o} \mathbf{v}\right|^{2} d s
$$

The associated scalar product is

$$
\left(\gamma_{o} \mathbf{u}, \gamma_{o} \mathbf{v}\right)_{\Gamma}=\int_{\Gamma} \sigma(x) \gamma_{o} \mathbf{u} \cdot \gamma_{o} \mathbf{v} d s
$$

According to assumption (16) this is equivalent to the usual $\mathrm{L}^{2}$ metric. It is assumed that the function $\sigma \in$ $C^{\infty}(\Gamma)$.

(9) For the purpose of stability and uniqueness we define the following norm:

$$
\int_{\Gamma} \eta d s=\|\eta\|_{0, \Gamma}
$$

(10) We shall deal extensively with the energy associated with fluids of second grade defined for the purpose of this study by

$$
\widetilde{E}_{v}=\frac{\alpha}{2}\|\mathbf{A}(\mathbf{v})\|_{\Omega}^{2}+\rho\|\mathbf{v}\|_{\Omega}^{2}+C_{1}\|\eta\|_{0, \Gamma}^{2}+C_{2}\left\|\nabla_{s} \eta\right\|_{0, \Gamma}^{2}
$$

with $C_{1}=(\delta \rho-2 \alpha K)$ and $C_{2}=\alpha \delta . \widetilde{E}_{v}^{1 / 2}$ is evidently a norm on $\mathbf{H}_{\zeta}^{1}(\Omega)$. We shall refer to the quantity $\widetilde{E}_{v}^{1 / 2}$ as the energy norm of $\mathbf{v}$.

(11) The constant $C$, which appears in inequalities, denotes a generic positive constant. Sometimes it is necessary to indicate the quantities on which a constant depends in brackets or by a subscript.

\subsection{Important Identities}

Identity 1. For any symmetric tensor $\mathbf{A}$ and any arbitrary tensor $\mathbf{B}$, we have $\mathbf{A}: \mathbf{B}=\mathbf{A}: \mathbf{B}_{s}$, with $\mathbf{B}_{s}=(1 / 2)\left(\mathbf{B}+\mathbf{B}^{T}\right)$.
Proof. Consider the following:

$$
\mathbf{A}: \mathbf{B}=\mathbf{A}^{T}: \mathbf{B}^{T}=\mathbf{A}: \mathbf{B}^{T}=\frac{1}{2} \mathbf{A}\left(\mathbf{B}+\mathbf{B}^{T}\right)=\mathbf{A}: \mathbf{B}_{s} .
$$

Expressions are necessary for inner products of the form $\left(D_{t} \mathbf{F}, \mathbf{F}\right)_{\Omega}$, where $\mathbf{F}$ is either a vector or a second order tensor. $D_{t}=\partial_{t}+\mathbf{v} \cdot \nabla$ is the material derivative. In order to obtain simple expressions for the scalar product, we notice that if o denotes either the usual scalar product or the "colon" product, then

$$
\left[\partial_{t} \mathbf{F}+(\mathbf{v} \cdot \nabla) \mathbf{F}\right] \circ \mathbf{F}=\frac{1}{2} \partial_{t}|\mathbf{F}|^{2}+\frac{1}{2} \nabla \cdot\left(|\mathbf{F}|^{2} \mathbf{v}\right),
$$

provided $\nabla \cdot \mathbf{v}=0$. Hence the following identity.

Identity 2. For any smooth vector or tensor quantity $\mathbf{F}(x, t)$ and any $\mathbf{v} \in \mathscr{D}$, we have

$$
\left(D_{t} \mathbf{F}, \mathbf{F}\right)_{\Omega}=\frac{1}{2} \partial_{t}\|\mathbf{F}\|_{\Omega}^{2}-\frac{1}{2} \int_{\Gamma}|\mathbf{F}|^{2} \eta d s
$$

Proof. By the divergence theorem

$$
\begin{aligned}
\left(\partial_{t} \mathbf{F}+(\mathbf{v} \cdot \nabla) \mathbf{F}, \mathbf{F}\right) & =\frac{1}{2} \partial_{t} \int_{\Omega}|\mathbf{F}|^{2} d x+\frac{1}{2} \int_{\Omega} \nabla \cdot\left(|\mathbf{F}|^{2} \mathbf{v}\right) d x \\
& =\frac{1}{2} \partial_{t}\|\mathbf{F}\|_{\Omega}^{2}+\frac{1}{2} \int_{\Gamma}|\mathbf{F}|^{2} \mathbf{v} \cdot \mathbf{n} d s \\
& =\frac{1}{2} \partial_{t}\|\mathbf{F}\|_{\Omega}^{2}-\frac{1}{2} \int_{\Gamma}|\mathbf{F}|^{2} \eta d s
\end{aligned}
$$

Later in this study we shall employ the energy method. It will become necessary to use the various boundary conditions in order to prove stability. The following is important to obtain the required results.

Identity 3. If $f \in H^{1}(\Omega)$ is a scalar field and $\mathbf{v} \in \mathscr{D}$, then

$$
\int_{\Omega}(\mathbf{v} \cdot \nabla) f d x=-\int_{\Gamma} \eta f d s .
$$

Proof. Integrating by parts and using the fact that $\mathbf{v}$ is solenoidal

$$
\int_{\Omega}(\mathbf{v} \cdot \nabla) f d x=\int_{\Gamma} f \mathbf{v} \cdot \mathbf{n} d s-\int_{\Omega} f \nabla \cdot \mathbf{v} d x=-\int_{\Gamma} \eta f d s .
$$

We note that, in particular for $\mathbf{v} \in \mathscr{D}$, the imbedding of $\mathbf{H}^{2}(\Omega)$ in the space of bounded continuous functions makes the choice $f=|\mathbf{v}|^{2}$ possible, and it follows from Identity 3 that

$$
\int_{\Omega}(\mathbf{v} \cdot \nabla)|\mathbf{v}|^{2} d s=-\int_{\Gamma}|\eta|^{3} d s
$$


For $\mathbf{v} \in \mathscr{D}$ we may also choose $f=|\mathbf{A}(\mathbf{v})|^{2}$, and it follows that

$$
\begin{aligned}
\int_{\Omega}(\mathbf{v} \cdot \nabla)|\mathbf{A}(\mathbf{v})|^{2} d x & =-\int_{\Gamma}|\mathbf{A}(\mathbf{v})|^{2} \eta d s \\
& =-\int_{\Gamma} 4 \eta^{3}|\mathbf{M}|^{2} d s
\end{aligned}
$$

since $\mathbf{N}=0$ on $\mathscr{D}$.

The following will be of immediate importance.

Identity 4. For any $\mathbf{v} \in \mathscr{D}$,

$$
\|\mathbf{A}(\mathbf{v})\|_{\Omega}^{2}=2\|\nabla \mathbf{v}\|_{\Omega}^{2}+2 \int_{\Gamma} K(x) \eta^{2} d s .
$$

Proof. From the definition of $\mathbf{A}$ it is evident that $|\mathbf{A}(\mathbf{v})|^{2}=$ $2|\nabla \mathbf{v}|^{2}+2 \nabla \mathbf{v}: \nabla^{T} \mathbf{v}$. Now $\nabla \cdot[(\mathbf{v} \cdot \nabla) \mathbf{v}]=\nabla \mathbf{v}: \nabla^{T} \mathbf{v}+(\mathbf{v} \cdot \nabla)(\nabla \cdot \mathbf{v})$, and, since $\nabla \cdot \mathbf{v}=0, \nabla \mathbf{v}: \nabla^{T} \mathbf{v}=\nabla \cdot[(\mathbf{v} \cdot \nabla) \mathbf{v}]$, integration over $\Omega$ and Identity 1 yield

$$
\begin{aligned}
\|\mathbf{A}(\mathbf{v})\|_{\Omega}^{2} & =2\|\nabla \mathbf{v}\|_{\Omega}^{2}+2 \int_{\Omega} \nabla \cdot[(\mathbf{v} \cdot \nabla) \mathbf{v}] d x \\
& =2\|\nabla \mathbf{v}\|_{\Omega}^{2}+2 \int_{\Gamma} \mathbf{n} \cdot[(\mathbf{v} \cdot \nabla) \mathbf{v}] d s \\
& =2\|\nabla \mathbf{v}\|_{\Omega}^{2}-2 \int_{\Gamma} \eta \mathbf{n} \otimes \mathbf{n}:[\nabla \mathbf{v}] d s \\
& =2\|\nabla \mathbf{v}\|_{\Omega}^{2}-\int_{\Gamma} \eta \mathbf{n} \cdot \mathbf{A}(\mathbf{v}) \mathbf{n} d s \\
& =2\|\nabla \mathbf{v}\|_{\Omega}^{2}+2 \int_{\Gamma} K(x) \eta^{2} d s .
\end{aligned}
$$

Thus, if the curvature $K$ is positive everywhere on $\Gamma$, it becomes apparent that $\mathbf{A}(\mathbf{v})=\mathbf{0}$ if and only if $\mathbf{v}=0$.

Identity 5. For any bilinear form $b$ on a Hilbert space $H$, we have, for any $\mathbf{v}, \mathbf{w} \in H$ and with $\mathbf{u}=\mathbf{v}-\mathbf{w}$ that $b(\mathbf{v}, \mathbf{v})-$ $b(\mathbf{w}, \mathbf{w})=b(\mathbf{u}, \mathbf{v})+b(\mathbf{w}, \mathbf{u})$.

Identity 6. Let $\mathbf{f}$ and $\mathbf{g}$ be tensor fields of the same order and let - denote the "scalar product" in such tensor fields. For $\mathbf{v} \in \mathscr{D}$ it is true that

$$
\int_{\Omega}[\mathbf{f} \circ(\mathbf{v} \cdot \nabla) \mathbf{g}+\mathbf{g} \circ(\mathbf{v} \cdot \nabla) \mathbf{f}] d x=-\int_{\Gamma} \eta_{v} \mathbf{f} \circ \mathbf{g} d s .
$$

Proof. Consider the following:

$$
\int_{\Omega} \mathbf{f} \circ(\mathbf{v} \cdot \nabla) \mathbf{g} d x=\int_{\Gamma}(\mathbf{v} \cdot \mathbf{n}) \mathbf{f} \circ \mathbf{g} d s-\int_{\Omega} \mathbf{g} \circ(\mathbf{v} \cdot \nabla) \mathbf{f} d x
$$

thus

$$
\int_{\Omega}[\mathbf{f} \circ(\mathbf{v} \cdot \nabla) \mathbf{g}+\mathbf{g} \circ(\mathbf{v} \cdot \nabla) \mathbf{f}] d x=\int_{\Gamma} \eta_{\nu} \mathbf{f} \circ \mathbf{g} d s .
$$

\subsection{Inequalities}

Lemma 4. Under the assumptions (17) and (16), for $\mathbf{v} \in \mathscr{D}$, $\mathbf{v}=0$ if and only if $\mathbf{A}(\mathbf{v})=0$.

Proof. From (16), (78), and (17) we have

$$
\frac{g}{S}\|\eta\|_{\Gamma}^{2}+\|\nabla \mathbf{v}\|_{\Omega}^{2} \leq \frac{1}{2}\|\mathbf{A}(\mathbf{v})\|_{\Omega}^{2} \leq\|\nabla \mathbf{v}\|_{\Omega}^{2}+\frac{G}{s}\|\eta\|_{\Gamma}^{2}
$$

and the result follows.

The following two lemmas are important in establishing a Poincaré inequality.

Lemma 5. The bilinear forms $a(\mathbf{u}, \mathbf{v})=(\mathbf{A}(\mathbf{u}), \mathbf{A}(\mathbf{v}))_{\Omega}$ and $b(\mathbf{u}, \mathbf{v})=\rho(\mathbf{u}, \mathbf{v})_{\Omega}+C_{1}\left(\gamma_{o} \mathbf{u}, \gamma_{o} \mathbf{v}\right)_{\Gamma}+C_{2}\left(\nabla_{s} \eta_{u}, \nabla_{s} \eta_{v}\right)_{\Gamma}$ are bounded in the space $\mathbf{H}_{\varsigma}^{1}(\Omega) . C_{1}$ and $C_{2}$ are positive constants.

Proof. For $\mathbf{u}$ and $\mathbf{v} \in \mathbf{H}_{\varsigma}^{1}(\Omega)$ and by (16), (17), and the Schwartz inequality

$$
\begin{aligned}
|a(\mathbf{u}, \mathbf{v})| & =|(\mathbf{A}(\mathbf{u}), \mathbf{A}(\mathbf{v}))| \\
& =\left|2(\nabla \mathbf{u}, \nabla \mathbf{v})_{\Omega}+2 \int_{\Gamma} K(x) \eta_{u} \eta_{v} d s\right| \\
& \leq 2\|\mathbf{u}\|_{1}\|\mathbf{v}\|_{1}+\frac{2 G}{s^{2}}\left\|\gamma_{o} \mathbf{u}\right\|_{\Gamma}\left\|\gamma_{o} \mathbf{v}\right\|_{\Gamma} .
\end{aligned}
$$

Hence, by the Trace theorem

$$
|a(\mathbf{u}, \mathbf{v})| \leq C\|\mathbf{u}\|_{1}\|\mathbf{v}\|_{1}
$$

Furthermore,

$$
\begin{aligned}
|b(\mathbf{u}, \mathbf{v})|= & \left|\rho(\mathbf{u}, \mathbf{v})_{\Omega}+C_{1}\left(\gamma_{o} \mathbf{u}, \gamma_{o} \mathbf{v}\right)_{\Gamma}+C_{2}\left(\nabla_{s} \eta_{u}, \nabla_{s} \eta_{u}\right)_{\Gamma}\right| \\
\leq & \rho\|\mathbf{u}\|_{1}\|\mathbf{v}\|_{1}+C_{1}\left\|\gamma_{o} \mathbf{u}\right\|_{\Gamma}\left\|\gamma_{o} \mathbf{v}\right\|_{\Gamma} \\
& +C_{2}\left\|\nabla_{s} \eta_{u}\right\|_{\Gamma}\left\|\nabla_{s} \eta_{v}\right\|_{\Gamma} \\
\leq & C\|\mathbf{u}\|_{1}\|\mathbf{v}\|_{1},
\end{aligned}
$$

by the Trace theorem and $C_{1}$ and $C_{2}$ as defined and $C$ generic.

Lemma 6. The bilinear form $|a(\mathbf{u}, \mathbf{v})|=(\mathbf{A}(\mathbf{u}), \mathbf{A}(\mathbf{v}))_{\Omega}$ is coercive in the sense that there exist constants $c_{1}>0$ and $c_{o} \geq 0$ such that

$$
|a(\mathbf{u}, \mathbf{u})| \geq c_{1}\|\mathbf{u}\|_{1}^{2}-c_{o} b(\mathbf{u}, \mathbf{u}) .
$$

Proof. From (83) we have

$$
\begin{aligned}
a(\mathbf{u}, \mathbf{u}) & =(\mathbf{A}(\mathbf{u}), \mathbf{A}(\mathbf{u}))_{\Omega} \\
& \geq 2\|\nabla \mathbf{u}\|_{\Omega}^{2}+\frac{2 g}{S}\|\eta\|_{\Gamma}^{2} \\
& =2\|\mathbf{u}\|_{1}^{2}-2\|\mathbf{u}\|_{\Omega}^{2}+\frac{2 g}{S}\|\eta\|_{\Gamma}^{2}
\end{aligned}
$$




$$
\begin{aligned}
& \geq 2\|\mathbf{u}\|_{1}^{2}-\frac{2}{\rho}\left(\rho\|\mathbf{u}\|_{\Omega}^{2}\right)-\frac{2}{\rho} C_{1}\|\eta\|_{\Gamma}^{2}-\frac{2}{\rho} C_{2}\left\|\nabla_{s} \eta_{u}\right\|_{\Gamma}^{2} \\
& =2\|\mathbf{u}\|_{1}^{2}-\frac{2}{\rho} b(\mathbf{u}, \mathbf{u}) .
\end{aligned}
$$

We can now obtain a generalised Poincaré inequality.

Lemma 7. There exists a smallest possible constant $\beta>0$ such that, for every $\mathbf{v} \in \mathbf{H}_{\varsigma}^{1}(\Omega)$,

$$
\frac{\beta}{2}\|\mathbf{A}(\mathbf{v})\|_{\Omega}^{2} \geq \rho\|\mathbf{v}\|_{\Omega}^{2}+C_{1}\left\|\gamma_{o} \mathbf{v}\right\|_{\Gamma}^{2}+C_{2}\left\|\nabla_{s} \eta_{u}\right\|_{\Gamma}^{2} .
$$

Proof. From the smoothness of $\Gamma$ (which is always assumed), it follows that the embedding $J: \mathbf{u} \in \mathbf{H}^{1}(\Omega) \rightarrow\left\langle\mathbf{u}, \gamma_{0} \mathbf{u}\right\rangle \in$ $\mathbf{L}^{2}(\Omega) \times \mathbf{L}^{2}(\Gamma)$ is compact [40]. From the boundedness and coerciveness proved above it follows that there exists a smallest eigenvalue $\lambda$ and associated eigenfunction $\mathbf{u} \epsilon$ $\mathbf{H}_{\varsigma}^{1}(\Omega)$ for which $b(\mathbf{u}, \mathbf{u})=1$ (see [34]):

$$
\lambda=\inf \left\{a(\mathbf{v}, \mathbf{v}): \mathbf{v} \in \mathbf{H}_{\varsigma}^{1}(\Omega) ; b(\mathbf{v}, \mathbf{v})=1\right\}=a(\mathbf{u}, \mathbf{u})
$$

$\lambda>0$, for if it is zero, it follows that $\mathbf{u}=0$, which cannot be. It follows from (90) that for any $\mathbf{v} \in \mathbf{H}_{\varsigma}^{1}(\Omega)$ the inequality

$$
a(\mathbf{v}, \mathbf{v}) \geq \lambda\left[\rho\|\mathbf{v}\|_{\Omega}^{2}+C_{1}\left\|\gamma_{o} \mathbf{v}\right\|_{\Gamma}^{2}+C_{2}\left\|\nabla_{s} \eta_{u}\right\|_{\Gamma}^{2}\right]
$$

holds and that $\lambda$ is the largest such constant. Finally, we set $\beta=2 / \lambda$.

Remark 8. It is now easy to see that $\|\mathbf{A}(\cdot)\|_{\Omega}$ is a norm on $\mathbf{H}_{\varsigma}^{1}(\Omega)$.

In fact, we have the following lemma.

Lemma 9. For all $\mathbf{v} \in \mathbf{H}_{\varsigma}^{1}(\Omega)$ we have

$$
\frac{\alpha}{2}\|\mathbf{A}(\mathbf{v})\|_{\Omega}^{2} \leq \widetilde{E}_{v} \leq \frac{\alpha+\beta+2}{2}\|\mathbf{A}(\mathbf{v})\|_{\Omega}^{2} .
$$

Proof. Add $(\alpha / 2)\|\mathbf{A}(\mathbf{v})\|_{\Omega}^{2}+C_{2}\left\|\nabla_{s} \eta\right\|_{0 \Gamma}^{2}$ to both sides of the inequality (89):

$$
\begin{aligned}
\widetilde{E}_{v} & =\frac{\alpha}{2}\|\mathbf{A}(\mathbf{v})\|_{\Omega}^{2}+\rho\|\mathbf{v}\|_{\Omega}^{2}+C_{1}\|\eta\|_{\Gamma}^{2}+C_{2}\left\|\nabla_{s} \eta\right\|_{0, \Gamma} \\
& \leq \frac{\alpha+\beta+2}{2}\|\mathbf{A}(\mathbf{v})\|_{\Omega}^{2} .
\end{aligned}
$$

From the definition of the energy norm it is clear that

$$
\frac{\alpha}{2}\|\mathbf{A}(\mathbf{v})\|_{\Omega}^{2} \leq \widetilde{E}_{v}
$$

and the result follows.

From Lemma 7 it is clear that these are the best estimates of this form.
Lemma 10. The norms $\|\mathbf{A}(\mathbf{v})\|_{\Omega}$ and $\widetilde{E}_{v}^{1 / 2}$ are equivalent to the norm in the Sobolev space $\mathbf{H}^{1}(\Omega)$.

Proof. From (83) and (89) it follows that

$$
\begin{aligned}
\|\mathbf{A}(\mathbf{v})\|_{\Omega}^{2} & \geq 2\|\nabla \mathbf{v}\|_{\Omega}^{2}, \\
\|\mathbf{A}(\mathbf{v})\|_{\Omega}^{2} & \geq \frac{2 \rho}{\beta}\|\mathbf{v}\|_{\Omega}^{2} .
\end{aligned}
$$

The addition of the two inequalities above yields

$$
\|\mathbf{A}(\mathbf{v})\|_{\Omega}^{2} \geq\|\nabla \mathbf{v}\|_{\Omega}^{2}+\frac{\rho}{\beta}\|\mathbf{v}\|_{\Omega}^{2} .
$$

Let $k=\min (1, \rho / \beta)$; then

$$
\|\mathbf{A}(\mathbf{v})\|_{\Omega}^{2} \geq k\|\mathbf{v}\|_{1}^{2} .
$$

Equation (83) yields

$$
\begin{aligned}
\|\mathbf{A}(\mathbf{v})\|_{\Omega}^{2} & \leq 2\|\nabla \mathbf{v}\|_{\Omega}^{2}+\frac{2 G}{s}\|\eta\|_{\Gamma}^{2} \\
& \leq 2\|\nabla \mathbf{v}\|_{\Omega}^{2}+2\|\mathbf{v}\|_{\Omega}^{2}+\frac{2 G}{s}\|\eta\|_{\Gamma}^{2},
\end{aligned}
$$

and from the Trace theorem it follows that

$$
\|\mathbf{A}(\mathbf{v})\|_{\Omega}^{2} \leq C\|\mathbf{v}\|_{1}^{2} .
$$

From (92) it is evident that the energy norm is equivalent to the norm $\|\mathbf{A}(\mathbf{v})\|_{\Omega}$.

Remark 11. From the above lemma we may claim from the embedding $\mathbf{H}^{1}(\Omega) \rightarrow \mathbf{L}^{3}(\Gamma)$, [40], that there exists a constant $\tau>0$ such that

$$
\int_{\Gamma}\left|\gamma_{o} \mathbf{v}\right|_{\Gamma}^{3} d s \leq \tau\|\mathbf{A}(\mathbf{v})\|_{\Omega}^{3} \quad \text { for every } \mathbf{v} \in \mathbf{H}_{\sigma}^{1}(\Omega) .
$$

7.4. Stability and Uniqueness for the Model Problem $\mathscr{A} . \mathbf{v} \in$ $\mathbf{H}^{3}(\Omega) \cap \mathscr{D}$ satisfies the system of evolution equations

$$
\begin{gathered}
D_{t}[\rho \mathbf{v}(x, t)]=\nabla \cdot \mathbf{T}(\rho, \mathbf{v}) \quad \text { in } \Omega \times(0, \infty) \\
\partial_{t}\left[\rho \eta-\alpha \Delta_{s} \eta\right]+\delta^{-1} \gamma_{o} p=\mu \Delta_{s} \eta \quad \text { at } \Gamma \times(0, \infty) \\
\gamma_{o}[\mathbf{A}(\mathbf{v})]=-2 \eta M \quad \text { at } \Gamma \times(0, \infty) .
\end{gathered}
$$

We now derive an energy identity for the solutions of (101). Take the $\mathbf{L}^{2}(\Omega)$, scalar product with $\mathbf{v}$ on both sides of $(101)_{1}$. This produces

$$
\begin{aligned}
\left(D_{t}(\rho \mathbf{v}), \mathbf{v}\right) & =\frac{\rho}{2} \partial_{t}\|\mathbf{v}\|_{\Omega}^{2}-\frac{\rho}{2} \int_{\Omega} \eta^{3} d s \\
& =(\nabla \cdot \mathbf{T}, \mathbf{v}) \\
& =\int_{\Gamma} \gamma_{o} \mathbf{v} \cdot \mathbf{T n} d s-(\mathbf{T}, \nabla \mathbf{v}) \\
& =-\int_{\Gamma} \eta \mathbf{n} \cdot \mathbf{T n} d s-\frac{1}{2} \int_{\Omega} \mathbf{T}: \mathbf{A} d x .
\end{aligned}
$$


According to the formulation of the original problem on the boundary where $s(\eta)=\mathbf{n} \cdot \mathbf{T n}$, we obtain

$$
\begin{aligned}
&-\int_{\Gamma} \eta \mathbf{n} \cdot \operatorname{Tn} d s \\
&=-\int_{\Gamma} \eta\left(-\gamma_{o} p-2 \mu \eta K-2 \alpha K \eta_{t}\right. \\
&\left.\quad+4 \alpha K_{G} \eta^{2}-\alpha \eta \Delta_{s} \eta-\ell(t)\right) d s .
\end{aligned}
$$

From $(101)_{2}$ we obtain

$$
\gamma_{o} p=-\delta \partial_{t}\left[\rho \eta-\alpha \Delta_{s} \eta\right]+\delta \mu \Delta_{s} \eta
$$

Substitute (104) into (103) to obtain

$$
\begin{aligned}
-\int_{\Gamma} \eta \mathbf{n} \cdot \mathbf{T n} d s= & -\partial_{t} \int_{\Gamma} \frac{\delta \rho}{2}|\eta|^{2} d s-\delta \alpha \partial_{t}\left\|\nabla_{s} \eta\right\|_{0, \Gamma}^{2} \\
& -\delta \mu\left\|\nabla_{s} \eta\right\|_{0, \Gamma}^{2} \\
& +2 \mu \delta \int_{\Gamma} K \eta^{2} d s+\partial_{t} \int_{\Gamma} \delta \alpha K|\eta|^{2} d s \\
& -4 \alpha \delta \int_{\Gamma} K_{G} \eta^{3} d s-\alpha \delta \int_{\Gamma} \eta\left|\nabla_{s} \eta\right|^{2} d s .
\end{aligned}
$$

Also

$$
-\frac{1}{2}(\mathbf{T}, \mathbf{A})_{\Omega}=-\frac{1}{2} \mu\|\mathbf{A}\|_{\Omega}^{2}-\frac{\alpha}{4} \partial_{t}\|\mathbf{A}\|_{\Omega}^{2}+\alpha \int_{\Gamma}|\mathbf{M}|^{2} \eta^{3} d s .
$$

Therefore the energy identity for Problem $\mathscr{A}$ is

$$
\begin{aligned}
\partial_{t}\left[\frac{\rho}{2}\|\mathbf{v}\|_{\Omega}^{2}+\frac{\alpha}{4}\|\mathbf{A}(\mathbf{v})\|_{\Omega}^{2}\right. \\
\left.\quad+\int_{\Gamma}\left(\frac{\rho \delta}{2}-\alpha K\right)|\eta|^{2} d s+\delta \alpha\left\|\nabla_{s} \eta\right\|_{0, \Gamma}^{2}\right] \\
=-\frac{1}{2} \mu\|\mathbf{A}(\mathbf{v})\|_{\Omega}^{2}+\alpha \int_{\Gamma}\left(|M|^{2}-4 \delta K_{G}\right)|\eta|^{3} d s \\
\quad-\delta \mu\left\|\nabla_{s} \eta\right\|_{\Gamma}^{2}+2 \mu \delta \int_{\Gamma} K|\eta|^{2} d s-\alpha \delta \int_{\Gamma} \eta\left|\nabla_{s} \eta\right|^{2} d s .
\end{aligned}
$$

Now we can define an energy norm for Problem $\mathscr{A}$ as follows:

$$
\begin{aligned}
\widetilde{E}_{v}(t)= & \rho\|\mathbf{v}\|_{\Omega}^{2}+\frac{\alpha}{2}\|\mathbf{A}(\mathbf{v})\|_{\Omega}^{2} \\
& +\int_{\Gamma}(\delta \rho-2 \alpha K)|\eta|^{2} d s+2 \delta \alpha\left\|\nabla_{s} \eta\right\|_{0, \Gamma}^{2} .
\end{aligned}
$$

Note that here we have to make the assumption that $\delta \rho-$ $2 \alpha K>0$, which gives us a restriction on $K$. We define a parameter

$$
p_{2}=\frac{\alpha K}{\delta \rho} .
$$

It is now clear that stability can only be proved under the assumption that $p_{2} \in(0,1 / 2)$.
The Poincaré inequality (see [39]) states that there exists a smallest constant $c$ such that $\|\eta\|_{0, \Gamma}^{2} \geq c\left\|\nabla_{s} \eta\right\|_{0, \Gamma}^{2}$. Applying the Schwartz inequality and the above Poincaré inequality, we obtain

$$
\begin{aligned}
\frac{d}{d t} \widetilde{E}_{v}(t) \leq & -\mu\|\mathbf{A}(\mathbf{v})\|_{\Omega}^{2}+2 \alpha\left(G^{2}+H^{2}+4 \delta G^{2}\right)\|\eta\|_{0, \Gamma}^{3} \\
& +2 \delta\left\|\nabla_{s} \eta\right\|_{0, \Gamma}^{2}\left(\mu+\alpha\|\eta\|_{0, \Gamma}\right)+4 \delta \mu G\|\eta\|_{0, \Gamma}^{2} \\
\leq & -\widetilde{E}_{v}\left[\mu-2 \alpha\left(G^{2}+H^{2}+2 \delta G^{2}-\delta\right) \widetilde{E}_{v}^{1 / 2}\right. \\
& -2 \delta \mu-4 \delta \mu G]
\end{aligned}
$$

With $2 \alpha\left(G^{2}+H^{2}+4 \delta G^{2}-\delta\right)=\epsilon^{*}$ and $\mu(1-2 \delta-4 \delta G)=\epsilon^{* *}$ we have

$$
\frac{d}{d t} \widetilde{E}_{v} \leq-\widetilde{E}_{v}\left[\epsilon^{* *} \mu-\epsilon^{*} \widetilde{E}_{v}^{1 / 2}\right]
$$

Theorem 12 (stability for problem $\mathscr{A}$ ). If $p_{2} \in(0,1 / 2)$ and $\widetilde{E}_{v}(0)<\left(\epsilon^{* *} \mu / \epsilon^{*}\right)^{2}$, then the energy $\widetilde{E}_{v}(t)$ decreases exponentially to zero as $t \rightarrow \infty$.

The uniqueness of the solution of Problem $\mathscr{A}$ is treated in the same way as the uniqueness of the solution of the original problem (see [33]).

\section{Conclusion}

An extensive study was conducted to find expressions for the stress tensors of Newtonian and non-Newtonian fluids at a permeable surface. We employed the Serret-Frenet formulae exactly for this reason. Stability of the rest state and uniqueness were proven for a special case where a shear flow was taken into account.

These results proved to be valuable in applications for the study of blood flow, where they were applied to model the permeability of special capillaries in the formation of cerebrospinal fluid $[41,42]$. Here the authors have presented a mathematical model of the flow of blood through the permeable boundary of a blocked choroidal capillary in which the parameters could be controlled. The blood plasma was modelled as a Newtonian fluid and the nonlinear Stokes equations were supplemented with a boundary condition at the permeable interface of the specialized capillary. The existence of a unique weak solution, which depends on the viscosity and the nature of the curvature of the capillary, was proved. By incorporating in this model all the ultrafiltration parameters, which are presented in [41, 42], the authors have attempted (within the prescribed morphological and physiological properties of the microvascular environment) to adapt the model used by Maritz and Sauer [33] to reallife situations. Further applications could be found in the modelling of other permeable systems in the human body like the lymphatic glands and the urinary system.

With this research the authors have tried to prepare the ground for the applications of these results in the exploring of permeable surfaces in biosciences, engineering, and the natural sciences. The open question regarding the existence 
of a classical solution for the system (101) will be addressed in further research.

\section{Conflict of Interests}

The authors declare that there is no conflict of interests regarding the publication of this paper.

\section{References}

[1] J. Leray, "Etude de diverses équations intégrales nonlinéaires que pose hýdrodynamique," Journal de Mathématiques Pures et Appliquées, vol. 12, pp. 1-82, 1933.

[2] J. Leray, "Essai sur les mouvements plans d'un liquide visqueux que limitent des parois," Journal de Mathématiques Pures et Appliquées, vol. 13, pp. 331-418, 1934.

[3] J. Leray, "Sur le mouvement d'un liquide visqueux emplissant l'espace," Acta Mathematica, vol. 63, no. 1, pp. 193-248, 1934.

[4] E. Hopf, "Über die Anfangswertaufgabe für die hydrodynamischen Grundgleichungen," Mathematische Nachrichten, vol. 4, pp. 213-231, 1951.

[5] G. P. Galdi, M. Padula, and K. R. Rajagopal, "On the conditional stability of the rest state of a fluid of second grade in unbounded domains," Archive for Rational Mechanics and Analysis, vol. 109, no. 2, pp. 173-182, 1990.

[6] G. P. Galdi, "Mathematical theory of second-grade fluids," in Stability and Wave Propagation in Fluids and Solids, pp. 67-104, Springer, Berlin, Germany, 1995.

[7] S. N. Alekseenko, "Existence and asymptotic representation of weak solutions to the flowing problem under the condition of regular slippage on solid walls," Siberian Mathematical Journal, vol. 35, no. 2, pp. 209-230, 1994.

[8] D. Cioranescu and O. El Hacène, "Existence and uniqueness for fluids of second grade," in Nonlinear Partial Differential Equations and Their Applications, vol. 109 of Research Notes in Mathematics, pp. 178-197, Pitman, Boston, Mass, USA, 1984.

[9] D. Cioranescu and V. Girault, "Solutions variationnelles et classiques d'une famille de fluides de grade deux," Comptes Rendus de l'Académie des Sciences Series I, vol. 322, no. 12, pp. 1163-1168, 1996.

[10] D. Cioranescu and V. Girault, "Weak and classical solutions of a family of second grade fluids," International Journal of NonLinear Mechanics, vol. 32, no. 2, pp. 315-335, 1997.

[11] D. Cioranescu and E. H. Quazar, "Existence and uniqueness for fluids of second grade," in College de France Seminars, vol. 109 of Pitman Research Notes in Mathematics, pp. 178-197, Pitman, Boston, Mass, USA, 1984.

[12] V. Coscia and G. P. Galdi, "Existence, uniqueness and stability of regular steady motions of a second-grade fluid," International Journal of Non-Linear Mechanics, vol. 29, no. 4, pp. 493-506, 1994.

[13] G. P. Galdi, M. Grobbelaar-van Dalsen, and N. Sauer, "Existence and uniqueness of classical solutions of the equations of motion for second-grade fluids," Archive for Rational Mechanics and Analysis, vol. 124, no. 3, pp. 221-237, 1993.

[14] V. Coscia, A. Sequeira, and J. Videman, "Existence and uniqueness of classical solutions for a class of complexity 2 fluids," International Journal of Non-Linear Mechanics, vol. 30, no. 4, pp. 531-551, 1995.

[15] G. P. Galdi, M. Grobbelaar-Van Dalsen, and N. Sauer, "Existence and uniqueness of solutions of the equations of motion for a fluid of second grade with non-homogeneous boundary conditions," International Journal of Non-Linear Mechanics, vol. 30, no. 5, pp. 701-709, 1995.

[16] R. Berker, "Contrainte sur une paroi en contact avec un fluide visqueux classique, un fluide de Stokes, un fluide de ColemanNoll," Comptes Rendus de l'Académie des Sciences, vol. 258, pp. 5144-5147, 1964.

[17] P. Bourgin and J. A. Tichy, "The effect of an additional boundary condition on the plane creeping flow of a second-order fluid," International Journal of Non-Linear Mechanics, vol. 24, no. 6, pp. 561-569, 1989.

[18] C.-S. Man, "Nonsteady channel flow of ice as a modified secondorder fluid with power-law viscosity," Archive for Rational Mechanics and Analysis, vol. 119, no. 1, pp. 35-57, 1992.

[19] K. R. Rajagopal and A. S. Gupta, "An exact solution for the flow of a non-Newtonian fluid past an infinite porous plate," Meccanica, vol. 19, no. 2, pp. 158-160, 1984.

[20] K. R. Rajagopal and P. N. Kaloni, "Some remarks on boundary conditions for flows of fluids of the differential type," in Continuum Mechanics and its Applications, pp. 935-942, Hemisphere Press, 1989.

[21] K. R. Rajagopal, "On boundary conditions for fluids of the differential type," in Navier-Stokes Equations and Related NonLinear Problems, Plenum Press, 1995.

[22] R. L. Fosdick and K. R. Rajagopal, "Anomalous features in the model of 'second order fluids,' Archive for Rational Mechanics and Analysis, vol. 70, no. 2, pp. 145-152, 1979.

[23] R. L. Fosdick and K. R. Rajagopal, "Thermodynamics and stability of fluids of third grade," Proceedings of the Royal Society. London. Series A. Mathematical and Physical Sciences, vol. 339, no. 1738, pp. 351-377, 1980.

[24] I. Proudman, "An example of steady laminar flow at large Reynolds number," Journal of Fluid Mechanics, vol. 9, pp. 593$602,1960$.

[25] G. S. Beavers and D. D. Joseph, "Boundary conditions at a naturally permeable wall," Journal of Fluid Mechanics, vol. 30, no. 1, pp. 197-207, 1967.

[26] K. R. Rajagopal and L. Tao, Mechanics of Mixtures, vol. 35 of Advances in Mathematics for Applied Sciences, World Scientific, River Edge, NJ, USA, 1995.

[27] H. Lamb, Hydrodynamics, Cambridge Mathematical Library, Cambridge University Press, Cambridge, UK, 6th edition, 1932.

[28] O. A. Ladyzhenskaya, The Mathematical Theory of Viscous Incompressible Flow, Gordon and Breach, New York, NY, USA, 1969.

[29] N. Sauer, “On Helmholtz projections," in Navier-Stokes Equations: Theory and Numerical Methods, R. Salvi, Ed., vol. 388 of Pitman Research Notes in Maths Series, pp. 257-263, Longman, Harlow, UK, 1998.

[30] M. Schwartz, S. Green, and W. A. Rutledge, Vector Analysis: With Applications to Geometry and Physics, Harper's Mathematics Series, Harper \& Brothers, New York, NY, USA, 1960.

[31] A. J. M. Spencer, Continuum Mechanics, Longman Group Limited, London, UK, 1980.

[32] J. E. Dunn and R. L. Fosdick, “Thermodynamics, stability, and boundedness of fluids of complexity 2 and fluids of second grade," Archive for Rational Mechanics and Analysis, vol. 56, pp. 191-252, 1974.

[33] R. Maritz and N. Sauer, "On boundary permeation in NavierStokes and second grade incompressible fluids," Mathematical Models \& Methods in Applied Sciences, vol. 16, no. 1, pp. 59-75, 2006. 
[34] N. Sauer and A. van der Merwe, "Eigenvalue problems with the spectral parameter also in the boundary condition," Quaestiones Mathematicae, vol. 5, no. 1, pp. 1-27, 1982.

[35] C. E. Weatherburn, Differential Geometry of Three Dimensions, vol. 1, Cambridge University Press, 1931.

[36] C. E. Weatherburn, Differential Geometry of Three Dimensions, vol. 2, Cambridge University Press, New York, NY, USA, 1930.

[37] R. Maritz and N. Sauer, "Stability and uniqueness of second grade fluids in regions with permeable Boundaries," in Navier Stokes Equations and Related Non linear Problems, H. Amann, G. P. Galdi, K. Pileckas, and V. A. Solonnikov, Eds., pp. 153-164, VSP, 1998.

[38] C. Truesdell and W. Noll, "The non-linear field theories of mechanics," in Handbuch der Physik, S. Flügge, Ed., vol. 3, chapter 1, pp. 1-602, Springer, Berlin, Germany, 1965.

[39] J.-L. Lions and E. Magenes, Non-Homogeneous Boundary Value Problems and Applications, vol. 1, Springer, Berlin, Germany, 1972.

[40] R. A. Adams, Sobolev Spaces, Academic Press, 1975.

[41] R. Maritz and S. Faleye, "The formation of cerebrospinal fluid in the brain (the linearised model)," Advances and Applications in Mathematical Sciences, vol. 8, no. 1, pp. 39-60, 2011.

[42] R. Maritz, J. M. Munganga, and S. Faleye, "On boundary permeation through the walls of blocked cerebral capillaries: a non-linear model," Journal of Mathematical Fluid Mechanics, vol. 15, no. 3, pp. 567-581, 2013. 


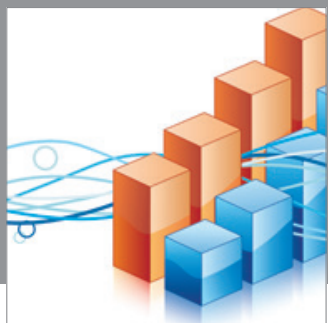

Advances in

Operations Research

mansans

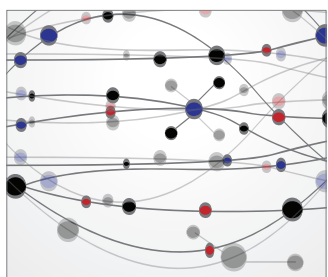

The Scientific World Journal
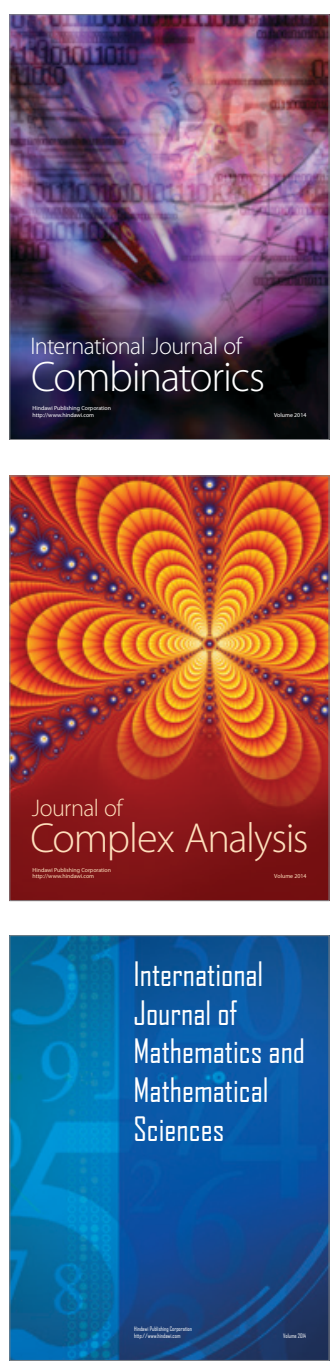
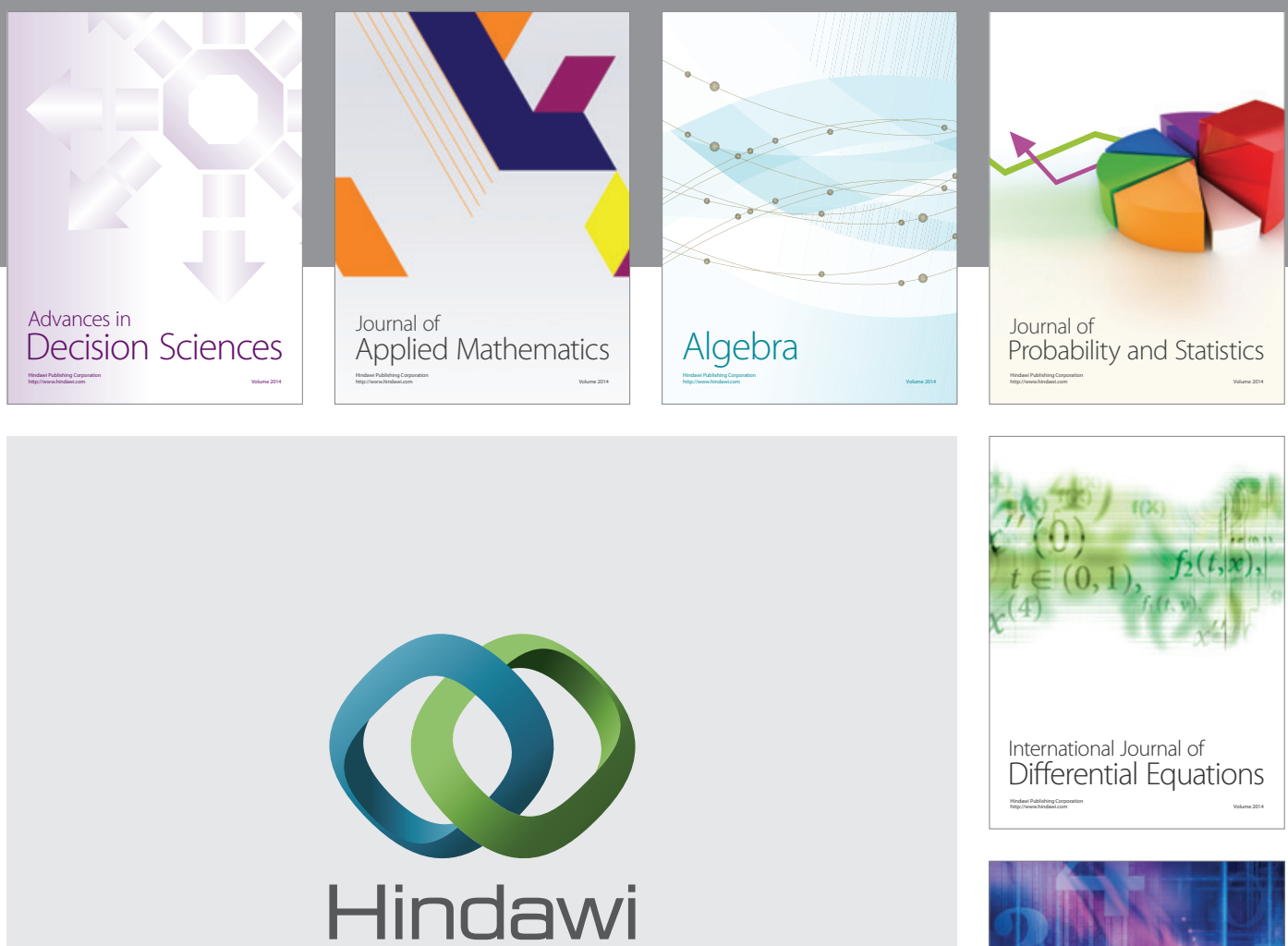

Submit your manuscripts at http://www.hindawi.com
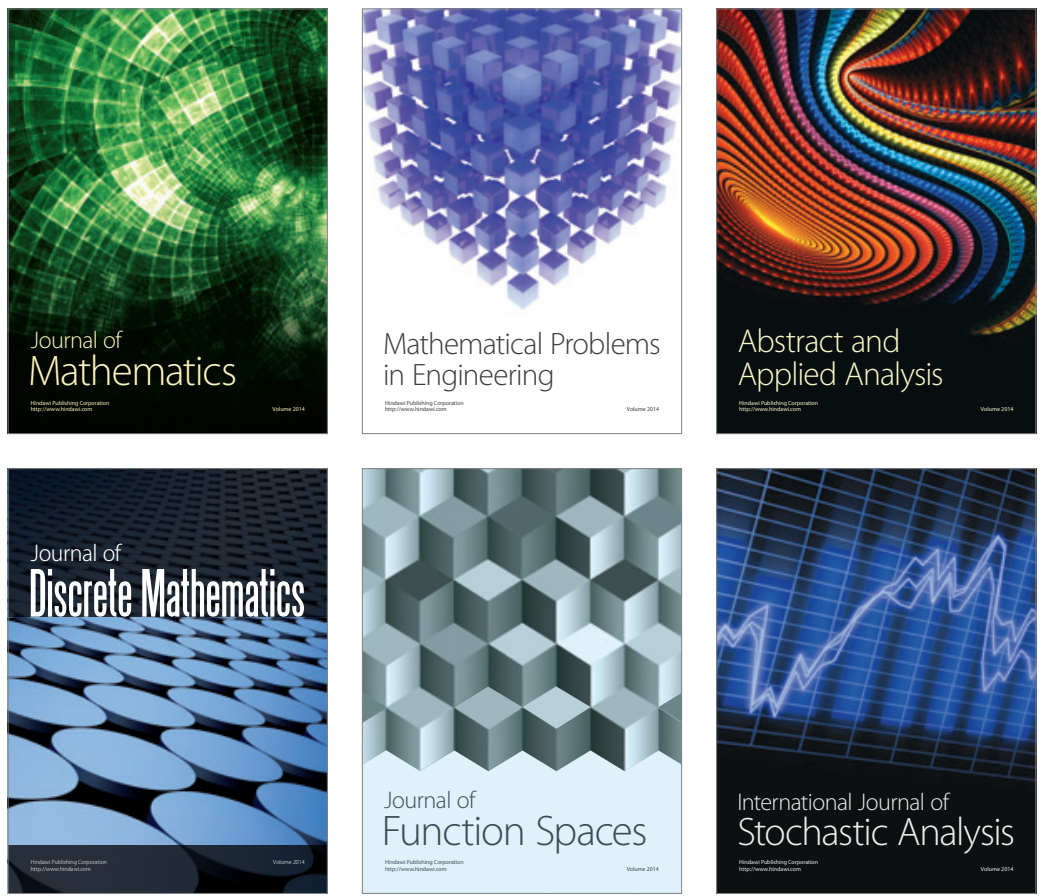

Journal of

Function Spaces

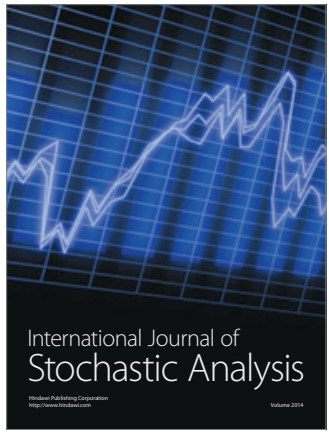

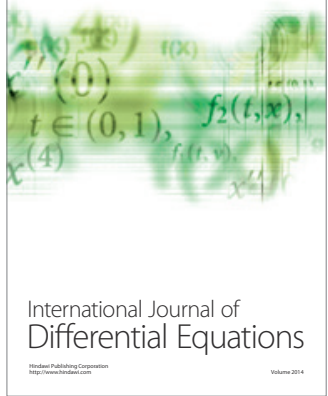
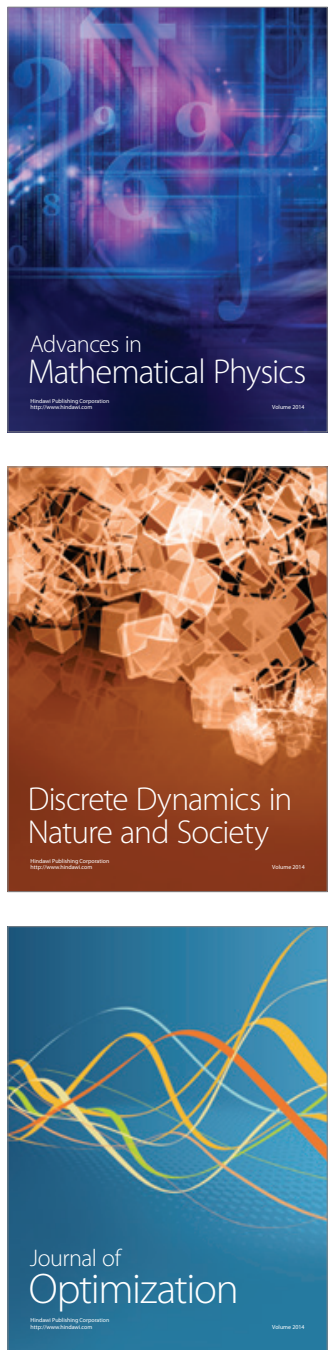